\title{
ERROR ESTIMATION OF THE BESSE RELAXATION SCHEME FOR A SEMILINEAR HEAT EQUATION
}

\author{
Georgios E. Zouraris*
}

\begin{abstract}
The solution to the initial and Dirichlet boundary value problem for a semilinear, one dimensional heat equation is approximated by a numerical method that combines the Besse Relaxation Scheme in time [C. R. Acad. Sci. Paris Sér. I 326 (1998)] with a central finite difference method in space. A new, composite stability argument is developed, leading to an optimal, second-order error estimate in the discrete $L_{t}^{\infty}\left(H_{x}^{2}\right)$-norm at the time-nodes and in the discrete $L_{t}^{\infty}\left(H_{x}^{1}\right)$-norm at the intermediate time-nodes. It is the first time in the literature where the Besse Relaxation Scheme is applied and analysed in the context of parabolic equations.
\end{abstract}

Mathematics Subject Classification. 65M12, 65M60.

Received February 14, 2019. Accepted November 10, 2020.

\section{INTRODUCTION}

\subsection{The initial boundary value problem}

Let $T>0, x_{a}, x_{b} \in \mathbb{R}$ with $x_{b}>x_{a}, \mathcal{I}:=\left[x_{a}, x_{b}\right], Q=[0, T] \times \mathcal{I}, g: \mathbb{R} \rightarrow \mathbb{R}, u_{0}: \mathcal{I} \rightarrow \mathbb{R}, f: Q \rightarrow \mathbb{R}$ and $u: Q \rightarrow \mathbb{R}$ be the solution of the following initial and boundary value problem:

$$
\begin{aligned}
u_{t} & =u_{x x}+g(u) u+f & & \text { on } Q, \\
u\left(t, x_{a}\right) & =u\left(t, x_{b}\right)=0 & & \forall t \in[0, T], \\
u(0, x) & =u_{0}(x) & & \forall x \in \mathcal{I} .
\end{aligned}
$$

Letting $\mu \in C^{\alpha}(\mathbb{R})$ with $\mu(x)=g(x) x$ and $f \in C_{t, x}^{\frac{\alpha}{2}, \alpha}(Q)$ for any $\alpha \in(0,1)$, and $u_{0} \in C^{2}(\mathcal{I})$ with

$$
u_{0}\left(x_{a}\right)=u_{0}\left(x_{b}\right)=0, \quad u_{0}^{\prime \prime}\left(x_{a}\right)+f\left(0, x_{a}\right)=u_{0}^{\prime \prime}\left(x_{b}\right)+f\left(0, x_{b}\right)=0
$$

the classical theory for parabolic problems yields that there exists a (at least) local solution $u \in C_{t, x}^{1,2}(Q)$, and we can achieve additional smoothness for $u$ by requiring additional regularity for $f, \mu, u_{0}$ along with compatibility conditions between $u_{0}$ and $f$ (see, e.g., [12]).

\footnotetext{
Keywords and phrases. Besse Relaxation Scheme, semilinear heat equation, finite differences, Dirichlet boundary conditions, optimal order error estimates.

Division of Applied Mathematics: Differential Equations and Numerical Analysis, Department of Mathematics and Applied Mathematics, University of Crete, GR-700 13 Voutes Campus, Heraklion, Crete, Greece.

* Corresponding author: georgios.zouraris@uoc.gr
} 


\subsection{Formulation of the numerical method}

Let $\mathbb{N}$ be the set of all positive integers and $\mathrm{L}:=x_{b}-x_{a}$. For given $N \in \mathbb{N}$, we define a uniform partition of the time interval $[0, T]$ with time-step $\tau:=\frac{T}{N}$, nodes $t_{n}:=n \tau$ for $n=0, \ldots, N$, and intermediate nodes $t^{n+\frac{1}{2}}=t_{n}+\frac{\tau}{2}$ for $n=0, \ldots, N-1$. Also, for given $J \in \mathbb{N}$, we consider a uniform partition of $\mathcal{I}$ with mesh-width $h:=\frac{\mathrm{L}}{J+1}$ and nodes $x_{j}:=x_{a}+j h$ for $j=0, \ldots, J+1$. Then, we introduce the discrete spaces

$$
\mathrm{X}_{h}:=\left\{\left(v_{j}\right)_{j=0}^{J+1}: v_{j} \in \mathbb{R}, j=0, \ldots, J+1\right\} \text { and } \mathbf{X}_{h}^{\circ}:=\left\{\left(v_{j}\right)_{j=0}^{J+1} \in \mathbf{X}_{h}: v_{0}=v_{J+1}=0\right\},
$$

a discrete product operator $\otimes \cdot: \mathbf{X}_{h} \times \mathbf{X}_{h} \rightarrow \mathbf{X}_{h}$ by $(v \otimes w)_{j}=v_{j} w_{j}$ for $j=0, \ldots, J+1$ and $v, w \in \mathbf{X}_{h}$, and a discrete Laplacian operator $\Delta_{h}: \mathrm{X}_{h}^{\circ} \rightarrow \mathrm{X}_{h}^{\circ}$ by $\Delta_{h} v_{j}:=\frac{v_{j-1}-2 v_{j}+v_{j+1}}{h^{2}}$ for $j=1, \ldots, J$ and $v \in \mathrm{X}_{h}^{\circ}$. In addition, we introduce operators $\mathrm{I}_{h}: C(\mathcal{I}) \rightarrow \mathrm{X}_{h}$ and $\mathrm{I}_{h}^{\circ}: C(\mathcal{I}) \rightarrow \mathrm{X}_{h}^{\circ}$, which, for given $z \in C(\mathcal{I})$, are defined by $\left(\mathrm{I}_{h} z\right)_{j}:=z\left(x_{j}\right)$ for $j=0, \ldots, J+1$ and $\left(\mathrm{I}_{h}^{\circ} z\right)_{j}:=z\left(x_{j}\right)$ for $j=1, \ldots, J$. Finally, for $q: \mathbb{R} \rightarrow \mathbb{R}$ and for any $w \in \mathrm{X}_{h}$, we define $q(w) \in \mathrm{X}_{h}$ by $(q(w))_{j}:=q\left(w_{j}\right)$ for $j=0, \ldots, J+1$.

The Besse Relaxation Finite Difference (BRFD) method combines a standard finite difference discretization in space with the Besse Relaxation Scheme in time (cf. [4]). Its algorithm consists of the following steps:

Step I. Define $U^{0} \in \mathrm{X}_{h}^{\circ}$ by

$$
U^{0}:=\mathrm{I}_{h}^{\circ}\left[u_{0}\right]
$$

and then find $U^{\frac{1}{2}} \in \mathrm{X}_{h}^{\circ}$ such that

$$
\frac{U^{\frac{1}{2}}-U^{0}}{(\tau / 2)}=\Delta_{h}\left(\frac{U^{\frac{1}{2}}+U^{0}}{2}\right)+g\left(u^{0}\right) \otimes\left(\frac{U^{\frac{1}{2}}+U^{0}}{2}\right)+\mathrm{I}_{h}^{\circ}\left[\frac{f\left(t^{\frac{1}{2}}, \cdot\right)+f\left(t_{0}, \cdot\right)}{2}\right] .
$$

Step II. Define $\Phi^{\frac{1}{2}} \in \mathrm{X}_{h}$ by

$$
\Phi^{\frac{1}{2}}:=g\left(U^{\frac{1}{2}}\right)
$$

and then find $U^{1} \in \mathrm{X}_{h}^{\circ}$ such that

$$
\frac{U^{1}-U^{0}}{\tau}=\Delta_{h}\left(\frac{U^{1}+U^{0}}{2}\right)+\Phi^{\frac{1}{2}} \otimes\left(\frac{U^{1}+U^{0}}{2}\right)+\mathrm{I}_{h}^{\circ}\left[\frac{f\left(t_{1}, \cdot\right)+f\left(t_{0}, \cdot\right)}{2}\right] .
$$

Step III. For $n=1, \ldots, N-1$, first define $\Phi^{n+\frac{1}{2}} \in \mathrm{X}_{h}$ by

$$
\Phi^{n+\frac{1}{2}}:=2 g\left(U^{n}\right)-\Phi^{n-\frac{1}{2}}
$$

and then find $U^{n+1} \in \mathrm{X}_{h}^{\circ}$ such that

$$
\frac{U^{n+1}-U^{n}}{\tau}=\Delta_{h}\left(\frac{U^{n+1}+U^{n}}{2}\right)+\Phi^{n+\frac{1}{2}} \otimes\left(\frac{U^{n+1}+U^{n}}{2}\right)+\mathrm{I}_{h}^{\circ}\left[\frac{f\left(t_{n+1}, \cdot\right)+f\left(t_{n}, \cdot\right)}{2}\right] .
$$

Remark 1.1. Here, by performing one step with the linearized version of the Crank-Nicolson method (1.6), we compute a second order approximation $U^{\frac{1}{2}}$ of $u\left(t^{\frac{1}{2}}, \cdot\right)$ and thus $\Phi^{\frac{1}{2}}=g\left(U^{\frac{1}{2}}\right)$ is a second order approximation of $g\left(u\left(t^{\frac{1}{2}}, \cdot\right)\right)$, that effects a second order convergence of $\Phi^{n+\frac{1}{2}}$ to $g\left(u\left(t^{n+\frac{1}{2}}, \cdot\right)\right)$ (see Thm. 4.6 and Tab. 1 in Sect. 5).

It is worth to note that, in the bibliography, the Relaxation Scheme is formulated along with the initial choice $\Phi^{\frac{1}{2}}:=g\left(u^{0}\right)$ (see, e.g., $\left.[4,5,10,11]\right)$, which is a first order in time approximation of $g\left(u\left(t^{\frac{1}{2}}, \cdot\right)\right)$ and results a first order in time convergence of $\Phi^{n+\frac{1}{2}}$ to $g\left(u\left(t^{n+\frac{1}{2}}, \cdot\right)\right)$ (see Thm. 4.7 and Tab. 2 in Sect. 5). However, in both cases, the method exhibits a second order convergence of $U^{n}$ to $u\left(t_{n}, \cdot\right)$ (see Thms. 4.6 and 4.7).

Remark 1.2. The Relaxation Scheme seems to be a special two-step method over the nodes and the intermediate nodes of the partition of the time interval. Indeed, the computation of $U^{n+1}$ requires the knowledge of the previous approximations $\Phi^{n+\frac{1}{2}}$ and $U^{n}$, and the computation of $\Phi^{n+\frac{1}{2}}$ is based on the knowledge of $U^{n}$ and $\Phi^{n-\frac{1}{2}}$.

Remark 1.3. The (BRFD) method requires, at every time step, the solution of a tridiagonal linear system of algebraic equations. The discussion on the well-posedness of the (BRFD) method is postponed until Theorems 4.6 and 4.7 . 


\subsection{Relation to the bibliography}

Two decades ago, for the discretization in time of the nonlinear Schrödinger (NLS) equation, C. Besse [4] introduced a new linear-implicit, conservative time-stepping method (called Relaxation Scheme (RS)) as an attempt to avoid the numerical solution of the nonlinear systems of algebraic equations that the application of the implicit Crank-Nicolson method yields. The (RS) combined with a finite element or a finite difference space discretization, is computationally efficient (see, e.g., $[3,8,11]$ ) and performs as a second order method (see, e.g., $[5,11]$ ). Later, C. Besse [5], analysing the (RS), as a semidiscrete in time method to approximate the solution to the Cauchy problem for the (NLS) equation with power non-linearity, provided its convergence under small final time $T$, without concluding a convergent rate with respect to the time-step. Oelz and Trabelsi [14] formulated a time-discrete version of the (RS) for the approximation of the solution to the Cauchy problem for a special nonlinear Schrödinger equation occurring in plasma physics, and then developed a convergence analysis analogous to that of [5], without, also, arriving at a conclusion on the order of convergence. Katsaounis and Kyza [10] first proposed a finite element version of the (RS) over a non uniform partition of the time interval, and then constructed an posteriori bound for the error only at the time-nodes, under the assumption that the proposed method has a second order convergence at the intermediate time nodes. At this point, we would like to observe, that the finite element version of the (RS) proposed in $[10,11]$ requires the solution of two linear systems of algebraic equations at every time-step, and thus its computational complexity is two times higher than that of the corresponding finite difference version of the (RS).

Independently of the present work [16], C. Besse et al. [6] focusing on the cubic NLS equation, completed the convergence analysis of [4] by a proper consistency argument and arrived at a error bound consisting of the error approximating $g\left(u\left(t^{\frac{1}{2}}, \cdot\right)\right)$ along with a second order, with respect to the time step, term. However, the latter error estimate fails to explain the second order, experimental convergence of the (RS) under the choice $g\left(u_{0}(\cdot)\right)$ as an initial approximation of $g\left(u\left(t^{\frac{1}{2}}, \cdot\right)\right)$ (see Rem. 1.1). In addition, the technique used in $[5,6]$ for the convergence analysis of the time-discrete (RS) is not suitable for the error estimation of a fully-discrete version of the (RS), because it is based on the derivation of a priori bounds of the (RS) time-discrete approximations in higher order Sobolev norms.

Also, independently of the present work [16], Li et al. [13] formulated a finite element version of the (RS) for the approximation of the solution to the Cauchy problem for the one dimensional, fractional, cubic (NLS) equation and presented an error analysis based on the energy technique. However, the convergence proof contains a gap, which, unfortunately, can not be resolved within the energy method, because the (RS) has the soul of a multistep method and within the context of the (NLS) equation this can not be overcame (see, e.g., $[5,17])$.

The aim of the present work is to propose (RS) as an alternative mechanism to derive second order, linearly implicit methods for parabolic problems with a non linear structure. Our choice to consider a finite difference version of the (RS), rather than a finite element one, is due to its higher flexibility in deriving energy-type error estimates in the discrete $H^{1}$-norm, which is fundamental to avoid mesh conditions in the one space dimension case. Indeed, by building up a proper stability argument and using energy techniques based on the parabolic strong stability, we are able to prove an optimal, second order error estimate in a discrete $L_{t}^{\infty}\left(H_{x}^{1}\right)$-norm at the intermediate time nodes and in a discrete $L_{t}^{\infty}\left(H_{x}^{2}\right)$-norm at the time nodes, under the choice (1.7) for $\Phi^{\frac{1}{2}}$. Addressing the initial choice $\Phi^{\frac{1}{2}}=g\left(u^{0}\right)$, we arrive at a new second order error estimate in the discrete $L_{t}^{\infty}\left(H_{x}^{1}\right)$ norm at the time nodes. The convergence analysis we present here can be used as a guide in the development of error estimates for finite element versions of the (RS) and for finite difference versions of the (RS) in the higher space dimension case (see, e.g., [18]).

\subsection{An overview of the paper}

In the error analysis of the (BRFD) method, we face the locally Lipschitz nonlinearity of the problem by introducing the (MBRFD) scheme (see Sect. 4.2), which follows from the (BRFD) method after mollifying properly the terms with nonlinear structure $(c f .[1,9,15])$. The (MBRFD) approximations depend on a parameter $\delta>0$ and have the following key property: when their discrete $L^{\infty}$-norm is bounded by $\delta$, then they are also 
(BRFD) approximations, because, in that case, the molifier (see (4.1)) acts as an indentity. Assuming that $\delta$ is large enough and $\tau$ is sufficiently small, for the (BRFD) approximations, first we show that are well-defined (see Prop. 4.2), and then we establish an optimal, second order error estimate in the discrete $H^{2}$-norm at the time nodes and in the discrete $H^{1}$-norm at the intermediate time nodes (see Thm. 4.4). Letting $h$ and $\tau$ be sufficiently small (see (4.62)) and applying a discrete Sobolev inequality (see (2.2)), the latter convergence result implies that the discrete $L^{\infty}$-norm of the (MBRFD) approximations are lower than $\delta$ and thus they, also, are (BRFD) approximations. Finally, we show that the (BRFD) approximations are unique and hence inherit the convergence properties of the (MBRFD) scheme (see Thm. 4.6), i.e., there exist positive constants $C_{1}$ and $C_{2}$, independent of $\tau$ and $h$, such that

$$
\left|U^{\frac{1}{2}}-I_{h}^{\circ}\left[u\left(t^{\frac{1}{2}}, \cdot\right)\right]\right|_{1, h} \leq C_{1}\left(\tau^{2}+\sqrt{\tau} h^{2}\right)
$$

and

$$
\max _{0 \leq n \leq N-1}\left|\Phi^{n+\frac{1}{2}}-\mathrm{I}_{h}\left[g\left(u\left(t^{n+\frac{1}{2}}, \cdot\right)\right)\right]\right|_{1, h}+\max _{0 \leq n \leq N}\left|U^{n}-\mathrm{I}_{h}^{\circ}\left[u\left(t_{n}, \cdot\right)\right]\right|_{2, h} \leq C_{2}\left(\tau^{2}+h^{2}\right),
$$

where $|\cdot|_{1, h}$ is a discrete $H^{1}$-norm which is stronger than the discrete $L^{\infty}$-norm and $|\cdot|_{2, h}$ is a discrete $H^{2}$-norm (see Sect. 2). Also, we undertook the challenge to investigate the influence on the convergence rate of the initial choice $\Phi^{\frac{1}{2}}=g\left(u^{0}\right)$, which is a first order (with respect to $\tau$ ) approximation of $g\left(u\left(t^{\frac{1}{2}}, \cdot\right)\right)$ and it is used in actual computations by several authors (see, e.g., $[4,5,10,11]$ ). First, we show that the latter initial choice affects the order of convergence at the intermediate node (see Cor. 4.5), i.e., there exist a positive constant $C_{3}$, independent of $\tau$ and $h$, such that

$$
\max _{0 \leq n \leq N-1}\left|\Phi^{n+\frac{1}{2}}-\mathrm{I}_{h}\left[g\left(u\left(t^{n+\frac{1}{2}}, \cdot\right)\right)\right]\right|_{1, h} \leq C_{3}\left(\tau+h^{2}\right),
$$

which is confirmed by results from numerical experiments (see Sect. 5). Then, we show that the order of convergence at the time nodes is still optimal (see Thm. 4.7), i.e., there exist constants $C_{4}$, independent of $\tau$ and $h$, such that

$$
\max _{0 \leq n \leq N}\left[\left\|U^{n}-I_{h}^{\circ}\left[u\left(t_{n}, \cdot\right)\right]\right\|_{0, h}+\left|U^{n}-I_{h}^{\circ}\left[u\left(t_{n}, \cdot\right)\right]\right|_{1, h}\right] \leq C_{4}\left(\tau^{2}+h^{2}\right) .
$$

The result above is new and it is the first time in the bibliography that the observed second order experimental convergence of the Relaxation Scheme at the time nodes, under a first order in time approximation of $g\left(u\left(t^{\frac{1}{2}}, \cdot\right)\right)$, is mathematically explained.

We close this section by giving a brief overview of the paper. In Section 2, we introduce additional notation and provide a series of auxiliary results. Section 3 is dedicated to the estimation of several type of consistency errors and of the approximation error of a discrete elliptic projection. In Section 4, first we introduce a modified version of the (BRFD) method, and then we analyze its convergence properties and arrive at a set of conditions that ensure the well-posedness and convergence of the (BRFD) method. Finally, Section 5 contains results from numerical experiments confirming the outcome of the convergence analysis, and Section 6 contains some general conclusions of the work at hands.

\section{Preliminaries}

Let us introduce another discrete space by $\mathrm{G}_{h}:=\left\{\left(z_{j}\right)_{j=0}^{J}: z_{j} \in \mathbb{R}, \quad j=0, \ldots, J\right\}$ and the discrete space derivative operator $\delta_{h}: \mathrm{X}_{h} \rightarrow \mathrm{G}_{h}$ by $\delta_{h} v_{j}:=\frac{v_{j+1}-v_{j}}{h}$ for $j=0, \ldots, J$ and $v \in \mathbf{X}_{h}$. We provide $\mathrm{G}_{h}$ with an inner product $((\cdot, \cdot))_{0, h}$ defined by $((z, v))_{0, h}:=h \sum_{j=0}^{h} z_{j} v_{j}$ for $z, v \in \mathrm{G}_{h}$, and we shall denote by $\|\cdot\|_{0, h}$ the corresponding norm, i.e., $\|z\|_{0, h}:=\left[((z, z))_{0, h}\right]^{1 / 2}$ for $z \in \mathrm{G}_{h}$. Also, we provide $\mathrm{G}_{h}$ with a discrete maximum norm $\|\cdot\|_{\infty, h}$ defined by $\|v\|_{\infty, h}:=\max _{0 \leq j \leq J}\left|v_{j}\right|$ for $v \in \mathrm{G}_{h}$.

On $\mathrm{X}_{h}^{\circ}$ we define a discrete inner product $(\cdot, \cdot)_{0, h}$ by $(v, z)_{0, h}:=h \sum_{j=1}^{J} v_{j} z_{j}$ for $v, z \in \mathrm{X}_{h}^{\circ}$, and we shall denote by $\|\cdot\|_{0, h}$ its induced norm, i.e., $\|v\|_{0, h}:=\left[(v, v)_{0, h}\right]^{1 / 2}$ for $v \in \mathrm{X}_{h}^{\circ}$. Also, for $v \in C^{2}(\mathcal{I})$, we define a 
discrete elliptic projection $\mathrm{R}_{h}(v) \in \mathrm{X}_{h}^{\circ}$ of $v(c f .[2])$ by requiring

$$
\Delta_{h}\left(\mathrm{R}_{h}(v)\right)=\mathrm{I}_{h}^{\circ}\left(v^{\prime \prime}\right) .
$$

Moreover, we equip $\mathbf{X}_{h}$ with a discrete $L^{\infty}$-norm $|\cdot|_{\infty, h}$ defined by $|w|_{\infty, h}:=\max _{0 \leq j \leq J+1}\left|w_{j}\right|$ for $w \in \mathbf{X}_{h}$ and with a discrete $H^{1}$-seminorm $|\cdot|_{1, h}$ given by $|w|_{1, h}:=\left\|\delta_{h} w\right\|_{0, h}$ for $w \in \mathbf{X}_{h}$. It is easily seen that $|\cdot|_{1, h}$ becomes a norm when it is restricted on $\mathrm{X}_{h}^{\circ}$ and satisfies the following useful inequalities:

$$
\begin{aligned}
& |v|_{\infty, h} \leq \mathrm{L}^{1 / 2}|v|_{1, h} \quad \forall v \in \mathrm{X}_{h}^{\circ}, \\
& \|v\|_{0, h} \leq \mathrm{L}|v|_{1, h} \quad \forall v \in \mathrm{X}_{h}^{\circ} .
\end{aligned}
$$

Lemma 2.1. For all $v, z \in \mathrm{X}_{h}^{\circ}$ it holds that

$$
\begin{aligned}
\left(\Delta_{h} v, z\right)_{0, h} & =-\left(\delta_{h} v, \delta_{h} z\right)_{0, h}=\left(v, \Delta_{h} z\right)_{0, h}, \\
\left(\Delta_{h} v, v\right)_{h} & =-|v|_{1, h}^{2} \\
|v|_{1, h} & \leq \mathrm{L}\left\|\Delta_{h} v\right\|_{0, h} .
\end{aligned}
$$

Proof. Relations (2.4) and (2.5) are standard. The inequality (2.6) follows, easily, by combining (2.5), the Cauchy-Schwarz inequality and (2.3).

Thus, under the light of (2.6), we can provide $\mathrm{X}_{h}^{\circ}$ with a discrete $H^{2}$-norm $|\cdot|_{2, h}$ defined by $|v|_{2, h}:=\left\|\Delta_{h} v\right\|_{0, h}$ for $v \in \mathbf{X}_{h}^{\circ}$.

We close this section by some useful Lipschitz-type inequalities the proof of which, can be found in the appendix.

Lemma 2.2. Let $\mathfrak{g} \in C^{2}(\mathbb{R} ; \mathbb{R})$ with $\sup \left(\left|\mathfrak{g}^{\prime}\right|+\left|\mathfrak{g}^{\prime \prime}\right|\right)<+\infty$. Then, for $v, w \in \mathbf{X}_{h}^{\circ}$, it holds that

$$
|\mathfrak{g}(v)-\mathfrak{g}(w)|_{1, h} \leq \sup _{\mathbb{R}}\left|\mathfrak{g}^{\prime}\right||v-w|_{1, h}+\sup _{\mathbb{R}}\left|\mathfrak{g}^{\prime \prime}\right|\left\|\delta_{h} w\right\|_{\infty, h}\|v-w\|_{0, h}
$$

Proof. See Appendix A.

Lemma 2.3. Let $\mathfrak{g} \in C^{3}(\mathbb{R} ; \mathbb{R})$ with $\sup _{\mathbb{R}}\left(\left|\mathfrak{g}^{\prime}\right|+\left|\mathfrak{g}^{\prime \prime}\right|+\left|\mathfrak{g}^{\prime \prime \prime}\right|\right)<+\infty$. Then, for $v^{a}, v^{b}, z^{a}, z^{b} \in \mathrm{X}_{h}^{\circ}$, it holds that

$$
\begin{aligned}
\left\|\mathfrak{g}\left(v^{a}\right)-\mathfrak{g}\left(v^{b}\right)-\mathfrak{g}\left(z^{a}\right)+\mathfrak{g}\left(z^{b}\right)\right\|_{0, h} \leq & \sup _{\mathbb{R}}\left|\mathfrak{g}^{\prime \prime}\right|\left|z^{a}-z^{b}\right|_{\infty, h}\left\|v^{b}-z^{b}\right\|_{0, h} \\
& +\left(\sup _{\mathbb{R}}\left|\mathfrak{g}^{\prime}\right|+\sup _{\mathbb{R}}\left|\mathfrak{g}^{\prime \prime}\right|\left|z^{a}-z^{b}\right|_{\infty, h}\right)\left\|v^{a}-v^{b}-z^{a}+z^{b}\right\|_{0, h}
\end{aligned}
$$

and

$$
\begin{aligned}
\left|\mathfrak{g}\left(v^{a}\right)-\mathfrak{g}\left(v^{b}\right)-\mathfrak{g}\left(z^{a}\right)+\mathfrak{g}\left(z^{b}\right)\right|_{1, h} \leq & \mathcal{F}^{A}\left(v^{a}, v^{b}\right)\left|v^{a}-v^{b}-z^{a}+z^{b}\right|_{1, h} \\
& +\mathcal{F}^{B}\left(z^{a}, z^{b}\right)\left(\left\|v^{a}-v^{b}-z^{a}+z^{b}\right\|_{0, h}+\left\|v^{b}-z^{b}\right\|_{0, h}\right) \\
& +\mathcal{F}^{C}\left(z^{a}, z^{b}\right)\left(\left|v^{a}-v^{b}-z^{a}+z^{b}\right|_{1, h}+\left|v^{b}-z^{b}\right|_{1, h}\right),
\end{aligned}
$$

where: $\mathcal{F}^{A}\left(v^{a}, v^{b}\right):=\sup _{\mathbb{R}}\left|\mathfrak{g}^{\prime}\right|+\frac{\mathrm{L}^{1 / 2}}{2} \sup _{\mathbb{R}}\left|\mathfrak{g}^{\prime \prime}\right|\left(\left|v^{a}\right|_{1, h}+\left|v^{b}\right|_{1, h}\right), \mathcal{F}^{B}\left(z^{a}, z^{b}\right):=\sup _{\mathbb{R}}\left|\mathfrak{g}^{\prime \prime}\right|\left\|\delta_{h}\left(z^{a}-z^{b}\right)\right\| \|_{\infty, h}$ and $\mathcal{F}^{C}\left(z^{a}, z^{b}\right):=\left|z^{a}-z^{b}\right|_{\infty, h}^{\mathbb{R}}\left[\sup _{\mathbb{R}}\left|\mathfrak{g}^{\prime \prime}\right|+\stackrel{\mathbb{R}}{L} \sup _{\mathbb{R}}\left|\mathfrak{g}^{\prime \prime \prime}\right|\left(\left\|\delta_{h} z^{a}\right\|_{\infty, h}+\left\|\delta_{h} z^{b}\right\|_{\infty, h}\right)\right]$.

Proof. See Appendix B. 


\section{LOCAL ERRORS}

To simplify the notation, we set $t^{\frac{1}{4}}:=\frac{\tau}{4}, u^{\frac{1}{4}}:=\mathrm{I}_{h}\left[u\left(t^{\frac{1}{4}}, \cdot\right)\right], u^{n}:=\mathrm{I}_{h}\left[u\left(t_{n}, \cdot\right)\right]$ for $n=0, \ldots, N$, and $u^{n+\frac{1}{2}}:=$ $\mathrm{I}_{h}\left[u\left(t^{n+\frac{1}{2}}, \cdot\right)\right]$ for $n=0, \ldots, N-1, \zeta(t, x):=g(u(t, x))$ and $\xi(t, x):=g(u(t, x)) u(t, x)$. In view of the Dirichlet boundary conditions (1.2) and the compatibility conditions (1.4), it holds that $u^{\frac{1}{4}} \in \mathrm{X}_{h}^{\circ}, u^{n} \in \mathrm{X}_{h}^{\circ}$ for $n=0, \ldots, N$ and $u^{n+\frac{1}{2}} \in \mathrm{X}_{h}^{\circ}$ for $n=0, \ldots, N-1$.

\subsection{Time truncation error}

For $n=1, \ldots, N-1$, let $\mathrm{r}^{n} \in \mathrm{X}_{h}^{\circ}$ be determined by

$$
\frac{g\left(u^{n+\frac{1}{2}}\right)+g\left(u^{n-\frac{1}{2}}\right)}{2}=g\left(u^{n}\right)+\mathbf{r}^{n} .
$$

Also, let $r^{\frac{1}{4}} \in \mathrm{X}_{h}$ be defined by

$$
\frac{u^{\frac{1}{2}}-u^{0}}{(\tau / 2)}=\mathrm{I}_{h}\left[\frac{u_{x x}\left(t^{\frac{1}{2}}, \cdot\right)+u_{x x}\left(t_{0}, \cdot\right)}{2}\right]+g\left(u^{0}\right) \otimes\left(\frac{u^{\frac{1}{2}}+u^{0}}{2}\right)+\mathrm{I}_{h}\left[\frac{f\left(t^{\frac{1}{2}}, \cdot\right)+f\left(t_{0}, \cdot\right)}{2}\right]+\mathrm{r}^{\frac{1}{4}}
$$

and let $\mathrm{r}^{n+\frac{1}{2}} \in \mathrm{X}_{h}$ be specified by

$$
\frac{u^{n+1}-u^{n}}{\tau}=\mathrm{I}_{h}\left[\frac{u_{x x}\left(t_{n+1}, \cdot\right)+u_{x x}\left(t_{n}, \cdot\right)}{2}\right]+g\left(u^{n+\frac{1}{2}}\right) \otimes\left(\frac{u^{n+1}+u^{n}}{2}\right)+\mathrm{I}_{h}\left[\frac{f\left(t_{n+1}, \cdot\right)+f\left(t_{n}, \cdot\right)}{2}\right]+\mathrm{r}^{n+\frac{1}{2}}
$$

for $n=0, \ldots, N-1$. Using that $u \in C_{t, x}^{1,2}(Q)$ along with (1.1), (1.4) and the Dirichlet boundary conditions (1.2), we arrive at $u_{x x}(t, x)=-f(t, x)$ for $t \in[0, T]$ and $x \in\left\{x_{a}, x_{b}\right\}$. Thus, we have $\mathrm{r}^{\frac{1}{4}} \in \mathrm{X}_{h}^{\circ}$ and $\mathrm{r}^{n+\frac{1}{2}} \in \mathrm{X}_{h}^{\circ}$ for $n=0, \ldots, N-1$. Also, (3.2) and (3.3) along with (1.1), yield

$$
\mathrm{r}^{\frac{1}{4}}=\mathrm{r}_{A}^{\frac{1}{4}}-\mathrm{r}_{B}^{\frac{1}{4}} \quad \text { and } \quad \mathrm{r}^{n+\frac{1}{2}}=\mathrm{r}_{A}^{n+\frac{1}{2}}-\mathrm{r}_{B}^{n+\frac{1}{2}}, \quad n=0, \ldots, N-1,
$$

where $\mathrm{r}_{A}^{\frac{1}{4}}, \mathrm{r}_{B}^{\frac{1}{4}}, \mathrm{r}_{A}^{n+\frac{1}{2}}, \mathrm{r}_{B}^{n+\frac{1}{2}} \in \mathrm{X}_{h}^{\circ}$ be defined by

$$
\begin{aligned}
& \mathrm{r}_{A}^{\frac{1}{4}}:=\frac{u^{\frac{1}{2}}-u^{0}}{(\tau / 2)}-\mathrm{I}_{h}\left[u_{t}\left(t^{\frac{1}{4}}, \cdot\right)\right]-\mathrm{I}_{h}\left[\frac{u_{t}\left(t^{\frac{1}{2}}, \cdot\right)+u_{t}\left(t_{0}, \cdot\right)}{2}-u_{t}\left(t^{\frac{1}{4}}, \cdot\right)\right] \\
& \mathrm{r}_{B}^{\frac{1}{4}}:=-\left[g\left(u^{\frac{1}{4}}\right)-g\left(u^{0}\right)\right] \otimes \frac{u^{\frac{1}{2}}+u^{0}}{2}+g\left(u^{\frac{1}{4}}\right) \otimes\left[\frac{u^{\frac{1}{2}}+u^{0}}{2}-u^{\frac{1}{4}}\right]-\left[\frac{g\left(u^{\frac{1}{2}}\right) \otimes u^{\frac{1}{2}}+g\left(u^{0}\right) \otimes u^{0}}{2}-g\left(u^{\frac{1}{4}}\right) \otimes u^{\frac{1}{4}}\right]
\end{aligned}
$$

and

$$
\begin{aligned}
& \mathrm{r}_{A}^{n+\frac{1}{2}}:=\frac{u^{n+1}-u^{n}}{\tau}-\mathrm{I}_{h}\left[u_{t}\left(t^{n+\frac{1}{2}}, \cdot\right)\right]-\mathrm{I}_{h}\left[\frac{u_{t}\left(t_{n}, \cdot\right)+u_{t}\left(t_{n+1}, \cdot\right)}{2}-u_{t}\left(t^{n+\frac{1}{2}}, \cdot\right)\right], \\
& \mathrm{r}_{B}^{n+\frac{1}{2}}:=g\left(u^{n+\frac{1}{2}}\right) \otimes\left[\frac{u^{n+1}+u^{n}}{2}-u^{n+\frac{1}{2}}\right]-\left[\frac{g\left(u^{n+1}\right) \otimes u^{n+1}+g\left(u^{n}\right) \otimes u^{n}}{2}-g\left(u^{n+\frac{1}{2}}\right) \otimes u^{n+\frac{1}{2}}\right] .
\end{aligned}
$$

Applying the Taylor formula, with respect to $t$ around $t=t_{n}$, we obtain

$$
\mathrm{r}^{n}=\frac{\tau^{2}}{2} \mathrm{I}_{h}\left[\int_{0}^{\frac{1}{2}}\left[\left(\frac{1}{2}-s\right) \zeta_{t t}\left(t_{n}+s \tau, \cdot\right)+s \zeta_{t t}\left(t^{n-\frac{1}{2}}+s \tau, \cdot\right)\right] \mathrm{d} s\right], \quad n=1, \ldots, N-1 .
$$


Applying again the Taylor formula, with respect to $t$ around $t=t^{\frac{1}{4}}$, we have

$$
\begin{aligned}
\mathbf{r}_{A}^{\frac{1}{4}}= & \tau^{2} \mathbf{I}_{h}\left[\int_{0}^{\frac{1}{4}}\left[s^{2} u_{t t t}(s \tau, \cdot)+\left(\frac{1}{4}-s\right)^{2} u_{t t t}\left(t^{\frac{1}{4}}+s \tau, \cdot\right)\right] \mathrm{d} s\right] \\
& -\frac{\tau^{2}}{2} \mathbf{I}_{h}\left[\int_{0}^{\frac{1}{4}}\left[s u_{t t t}(s \tau, \cdot)+\left(\frac{1}{4}-s\right) u_{t t t}\left(t^{\frac{1}{4}}+s \tau, \cdot\right)\right] \mathrm{d} s\right], \\
\mathbf{r}_{B}^{\frac{1}{4}}= & -\tau \frac{u^{\frac{1}{2}}+u^{0}}{2} \otimes \mathbf{I}_{h}\left[\int_{0}^{\frac{1}{4}} \zeta_{t}(s \tau, \cdot) \mathrm{d} s\right] \\
& +\frac{\tau^{2}}{2} g\left(u^{\frac{1}{4}}\right) \otimes \mathbf{I}_{h}\left[\int_{0}^{\frac{1}{4}}\left[s u_{t t}(s \tau, \cdot)+\left(\frac{1}{4}-s\right) u_{t t}\left(t^{\frac{1}{4}}+s \tau, \cdot\right)\right] \mathrm{d} s\right] \\
& -\frac{\tau^{2}}{2} \mathbf{I}_{h}\left[\int_{0}^{\frac{1}{4}}\left[s \xi_{t t}(s \tau, \cdot)+\left(\frac{1}{4}-s\right) \xi_{t t}\left(t^{\frac{1}{4}}+s \tau, \cdot\right)\right] \mathrm{d} s\right] .
\end{aligned}
$$

Also, the Taylor formula, with respect to $t$ around $t=t^{n+\frac{1}{2}}$, yields

$$
\begin{aligned}
\mathrm{r}_{A}^{n+\frac{1}{2}}= & \frac{\tau^{2}}{2} \mathrm{I}_{h}\left[\int_{0}^{\frac{1}{2}}\left[s^{2} u_{t t t}\left(t_{n}+s \tau, \cdot\right)\right]+\left(\frac{1}{2}-s\right)^{2} u_{t t t}\left(t^{n+\frac{1}{2}}+s \tau, \cdot\right)\right] \mathrm{d} s \\
& -\frac{\tau^{2}}{2} \mathbf{I}_{h}\left[\int_{0}^{\frac{1}{2}}\left[s u_{t t t}\left(t_{n}+\tau s, \cdot\right)+\left(\frac{1}{2}-s\right) u_{t t t}\left(t^{n+\frac{1}{2}}+\tau s, \cdot\right)\right] \mathrm{d} s\right], \\
\mathbf{r}_{B}^{n+\frac{1}{2}}= & \frac{\tau^{2}}{2} g\left(u^{n+\frac{1}{2}}\right) \otimes \mathbf{I}_{h}\left[\int_{0}^{\frac{1}{2}}\left[s u_{t t}\left(t_{n}+s \tau, \cdot\right)+\left(\frac{1}{2}-s\right) u_{t t}\left(t^{n+\frac{1}{2}}+s \tau, \cdot\right)\right] \mathrm{d} s\right] \\
& -\frac{\tau^{2}}{2} \mathbf{I}_{h}\left[\int_{0}^{\frac{1}{2}}\left[s \xi_{t t}\left(t_{n}+\tau s, \cdot\right)+\left(\frac{1}{2}-s\right) \xi_{t t}\left(t^{n+\frac{1}{2}}+\tau s, \cdot\right)\right] \mathrm{d} s\right]
\end{aligned}
$$

for $n=0, \ldots, N-1$.

Thus, assuming that $g \in C^{4}(\mathbb{R}), u \in C_{t, x}^{3,0}(Q)$ and $\partial_{t}^{\ell} u \in C_{t, x}^{0,1}(Q)$ for $\ell=1,2,3$, from (3.4) to (3.7), we arrive at the following estimates:

$$
\begin{aligned}
\left\|\mathrm{r}_{A}^{\frac{1}{4}}\right\|_{0, h}+\tau\left\|\mathrm{r}_{B}^{\frac{1}{4}}\right\|_{0, h}+\max _{0 \leq n \leq N-1}\left\|\mathrm{r}^{n+\frac{1}{2}}\right\|_{0, h} & \leq \widehat{\mathrm{C}}_{1} \tau^{2} \max _{Q}\left(\left|u_{t t}\right|+\left|u_{t t t}\right|+\left|\zeta_{t}\right|+\left|\xi_{t t}\right|\right), \\
\max _{0 \leq n \leq N-1}\left|\mathrm{r}^{n+\frac{1}{2}}\right|_{1, h}+\tau\left|\mathrm{r}_{B}^{\frac{1}{4}}\right|_{1, h} & \leq \widehat{\mathrm{C}}_{2} \tau^{2} \max _{Q}\left(\left|u_{x t t}\right|+\left|u_{x t t t}\right|+\left|\zeta_{x t}\right|+\left|\xi_{x t t}\right|\right)
\end{aligned}
$$

and

$$
\begin{aligned}
& \max _{1 \leq n \leq N-1}\left\|\mathrm{r}^{n}\right\|_{0, h}+\max _{1 \leq n \leq N-1}\left|\mathrm{r}^{n}\right|_{1, h} \leq \widehat{\mathrm{C}}_{3} \tau^{2} \max _{Q}\left(\left|\zeta_{t t}\right|+\left|\zeta_{x t t}\right|\right), \\
& \max _{2 \leq n \leq N-1}\left\|\mathrm{r}^{n}-\mathrm{r}^{n-1}\right\|_{0, h}+\max _{2 \leq n \leq N-1}\left|\mathrm{r}^{n}-\mathrm{r}^{n-1}\right|_{1, h} \leq \widehat{\mathrm{C}}_{4} \tau^{3} \max _{Q}\left(\left|\zeta_{t t t}\right|+\left|\zeta_{x t t t}\right|\right) .
\end{aligned}
$$

\subsection{Space truncation error}

Also, let $\mathrm{s}^{\frac{1}{4}} \in \mathrm{X}_{h}^{\circ}$ be defined by

$$
\frac{u^{\frac{1}{2}}-u^{0}}{(\tau / 2)}=\Delta_{h}\left(\frac{u^{\frac{1}{2}}+u^{0}}{2}\right)+g\left(u^{0}\right) \otimes\left(\frac{u^{\frac{1}{2}}+u^{0}}{2}\right)+\mathrm{I}_{h}^{\circ}\left[\frac{f\left(t^{\frac{1}{2}}, \cdot\right)+f\left(t_{0}, \cdot\right)}{2}\right]+\mathrm{s}^{\frac{1}{4}}
$$


and, for $n=0, \ldots, N-1$, let $\mathrm{s}^{n+\frac{1}{2}} \in \mathrm{X}_{h}^{\circ}$ be given by

$$
\frac{u^{n+1}-u^{n}}{\tau}=\Delta_{h}\left(\frac{u^{n+1}+u^{n}}{2}\right)+g\left(u^{n+\frac{1}{2}}\right) \otimes\left(\frac{u^{n+1}+u^{n}}{2}\right)+I_{h}^{\circ}\left[\frac{f\left(t_{n+1}, \cdot\right)+f\left(t_{n}, \cdot\right)}{2}\right]+\mathrm{s}^{n+\frac{1}{2}} .
$$

Subtracting (3.12) from (3.2) and (3.13) from (3.3), we obtain

$$
\begin{aligned}
\mathrm{r}^{\frac{1}{4}}-\mathrm{s}^{\frac{1}{4}} & =\Delta_{h}\left(\frac{u^{\frac{1}{2}}+u^{0}}{2}\right)-\mathrm{I}_{h}^{\circ}\left[\frac{u_{x x}\left(t^{\frac{1}{2}}, \cdot\right)+u_{x x}\left(t_{0}, \cdot\right)}{2}\right], \\
\mathrm{r}^{n+\frac{1}{2}}-\mathrm{s}^{n+\frac{1}{2}} & =\Delta_{h}\left(\frac{u^{n+1}+u^{n}}{2}\right)-\mathrm{I}_{h}^{\circ}\left[\frac{u_{x x}\left(t_{n+1}, \cdot\right)+u_{x x}\left(t_{n}, \cdot\right)}{2}\right], \quad n=0, \ldots, N-1 .
\end{aligned}
$$

For $t \in[0, T]$, the use of the Taylor formula (with respect to $x$ around $x=x_{j}$ ) yields

$$
\begin{aligned}
\left(\Delta_{h}\left(\mathrm{I}_{h}^{\circ}[u(t, \cdot)]\right)-\mathrm{I}_{h}^{\circ}\left[u_{x x}(t, \cdot)\right]\right)_{j}= & \frac{h^{2}}{6} \int_{0}^{1}(1-y)^{3} u_{x x x x}\left(t, x_{j}+h y\right) \mathrm{d} y \\
& +\frac{h^{2}}{6} \int_{0}^{1} y^{3} u_{x x x x}\left(t, x_{j-1}+h y\right) \mathrm{d} y, \quad j=1, \ldots, J .
\end{aligned}
$$

Assuming that $u \in C_{t, x}^{0,4}(Q),(3.14)$ and (3.15) yield that

$$
\left\|\mathbf{s}^{\frac{1}{4}}-\mathrm{r}^{\frac{1}{4}}\right\|_{0, h}+\max _{0 \leq n \leq N-1}\left\|\mathbf{s}^{n+\frac{1}{2}}-\mathrm{r}^{n+\frac{1}{2}}\right\|_{0, h} \leq \frac{1}{12} h^{2} \max _{Q}\left|u_{x x x x}\right| .
$$

\subsection{Elliptic projection approximation error}

Let $v \in C^{4}(\mathcal{I})$. Using the Taylor formula $(c f .(3.15))$ it follows that

$$
\Delta_{h}\left(\mathrm{I}_{h}^{\circ}(v)\right)=\mathrm{I}_{h}^{\circ}\left(v^{\prime \prime}\right)+\frac{h^{2}}{6} \mathrm{r}^{\mathrm{EP}}(v)
$$

where $\mathrm{r}^{\mathrm{EP}}(v) \in \mathrm{X}_{h}^{\circ}$ is defined by

$$
\left(\mathrm{r}^{\mathrm{EP}}(v)\right)_{j}:=\int_{0}^{1}\left[(1-y)^{3} v^{\prime \prime \prime \prime}\left(x_{j}+h y\right)+y^{3} v^{\prime \prime \prime \prime}\left(x_{j-1}+h y\right)\right] \mathrm{d} y, \quad j=1, \ldots, J .
$$

Subtracting (2.1) from (3.17) we conclude that

$$
\Delta_{h}\left(\mathrm{I}_{h}^{\circ}(v)-\mathrm{R}_{h}(v)\right)=\frac{h^{2}}{6} \mathrm{r}^{\mathrm{EP}}(v),
$$

which, obviously, yields

$$
\left|\mathrm{R}_{h}(v)-\mathrm{I}_{h}^{\circ}(v)\right|_{2, h} \leq \frac{\sqrt{\mathrm{L}}}{12} h^{2} \max _{\mathcal{I}}\left|v^{\prime \prime \prime \prime}\right| .
$$

Also, combining (3.19) and (2.6), we obtain

$$
\left|\mathrm{R}_{h}(v)-\mathrm{I}_{h}^{\circ}(v)\right|_{1, h} \leq \frac{\mathrm{L}^{\frac{3}{2}}}{12} h^{2} \max _{\mathcal{I}}\left|v^{\prime \prime \prime \prime}\right| .
$$

We close this section with the following useful lemma.

Lemma 3.1. For $w \in C_{t, x}^{1,0}(Q)$ with $w_{t} \in C_{t, x}^{0,4}(Q)$, it holds that

$$
\left|\mathrm{R}_{h}\left[\frac{w(t, \cdot)-w(s, \cdot)}{t-s}\right]-\mathrm{I}_{h}^{\circ}\left[\frac{w(t, \cdot)-w(s, \cdot)}{t-s}\right]\right|_{1, h} \leq \frac{\mathrm{L}^{\frac{3}{2}}}{12} h^{2} \max _{Q}\left|w_{x x x x t}\right|
$$

for all $t, s \in[0, T]$ with $t>s$. 
Proof. Let $t, s \in[0, T]$ with $t>s$, and $\omega:=\mathrm{R}_{h}\left[\frac{w(t, \cdot)-w(s, \cdot)}{t-s}\right]-\mathrm{I}_{h}^{\circ}\left[\frac{w(t, \cdot)-w(s, \cdot)}{t-s}\right] \in \mathrm{X}_{h}^{\circ}$.

First, we observe that

$$
\begin{aligned}
|\omega|_{1, h} & =\frac{1}{t-s}\left|\int_{s}^{t}\left(\mathrm{R}_{h}\left[w_{t}\left(s^{\prime}, \cdot\right)\right]-\mathrm{I}_{h}^{\circ}\left[w_{t}\left(s^{\prime}, \cdot\right)\right]\right) \mathrm{d} s^{\prime}\right|_{1, h} \\
& \leq \frac{1}{t-s} \int_{s}^{t}\left|\mathrm{R}_{h}\left[w_{t}\left(s^{\prime}, \cdot\right)\right]-\mathrm{I}_{h}^{\circ}\left[w_{t}\left(s^{\prime}, \cdot\right)\right]\right|_{1, h} \mathrm{~d} s^{\prime},
\end{aligned}
$$

and then we use (3.20) to have

$$
\begin{aligned}
|\omega|_{1, h} & \leq \frac{\mathrm{L}^{\frac{3}{2}}}{12} \frac{h^{2}}{t-s}\left(\int_{s}^{t} \max _{\mathcal{I}}\left|w_{x x x x t}\left(s^{\prime}, \cdot\right)\right| \mathrm{d} s^{\prime}\right) \\
& \leq \frac{\mathrm{L}^{\frac{3}{2}}}{12} h^{2} \max _{Q}\left|w_{x x x x t}\right| .
\end{aligned}
$$

\section{Convergence Analysis}

\subsection{A mollifier}

For $\delta>0$, let $\mathfrak{n}_{\delta} \in C^{3}(\mathbb{R} ; \mathbb{R})(c f .[9,15])$ be an odd function defined by

$$
\mathfrak{n}_{\delta}(x):= \begin{cases}x, & \text { if } x \in[0, \delta], \\ p_{\delta}(x), & \text { if } x \in(\delta, 2 \delta], \quad \forall x \geq 0, \\ 2 \delta, & \text { if } x>2 \delta,\end{cases}
$$

where $p_{\delta}$ is the unique polynomial of $\mathbb{P}^{7}[\delta, 2 \delta]$ that satisfies the following conditions:

$$
p_{\delta}(\delta)=\delta, \quad p_{\delta}^{\prime}(\delta)=1, \quad p_{\delta}^{\prime \prime}(\delta)=p_{\delta}^{\prime \prime \prime}(\delta)=0, p_{\delta}(2 \delta)=2 \delta, \quad p_{\delta}^{\prime}(2 \delta)=p_{\delta}^{\prime \prime}(2 \delta)=p_{\delta}^{\prime \prime \prime}(2 \delta)=0 .
$$

\subsection{The (MBRFD) scheme}

The modified version of the (BRFD) method $(c f .[1,9,15])$ is a recursive procedure that, for given $\delta>0$, derives approximations $\left(V_{\delta}^{n}\right)_{n=0}^{N} \subset \mathrm{X}_{h}^{\circ}$ of the solution $u$ by performing the steps below:

Step 1. Let $V_{\delta}^{0} \in \mathrm{X}_{h}^{\circ}$ be defined by

$$
V_{\delta}^{0}:=u^{0}
$$

and $V_{\delta}^{\frac{1}{2}} \in \mathrm{X}_{h}^{\circ}$ be specified by

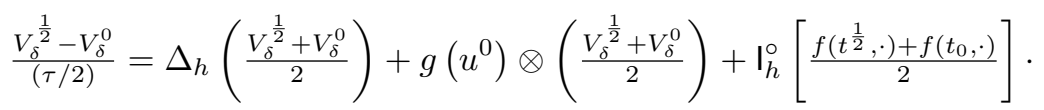

Step 2. Define $\Phi_{\delta}^{\frac{1}{2}} \in \mathrm{X}_{h}$ by

$$
\Phi_{\delta}^{\frac{1}{2}}:=g\left(\mathfrak{n}_{\delta}\left(V_{\delta}^{\frac{1}{2}}\right)\right)
$$

and find $V_{\delta}^{1} \in \mathrm{X}_{h}^{\circ}$ such that

$$
\frac{V_{\delta}^{1}-V_{\delta}^{0}}{\tau}=\Delta_{h}\left(\frac{V_{\delta}^{1}+V_{\delta}^{0}}{2}\right)+\mathfrak{n}_{\delta}\left(\Phi_{\delta}^{\frac{1}{2}}\right) \otimes\left(\frac{V_{\delta}^{1}+V_{\delta}^{0}}{2}\right)+\mathrm{I}_{h}^{\circ}\left[\frac{f\left(t_{1}, \cdot\right)+f\left(t_{0}, \cdot\right)}{2}\right] .
$$


Step 3. For $n=1, \ldots, N-1$, first define $\Phi_{\delta}^{n+\frac{1}{2}} \in \mathrm{X}_{h}$ by

$$
\Phi_{\delta}^{n+\frac{1}{2}}:=2 g\left(\mathfrak{n}_{\delta}\left(V_{\delta}^{n}\right)\right)-\Phi_{\delta}^{n-\frac{1}{2}}
$$

and, then, find $V_{\delta}^{n+1} \in \mathrm{X}_{h}^{\circ}$ such that

$$
\frac{V_{\delta}^{n+1}-V_{\delta}^{n}}{\tau}=\Delta_{h}\left(\frac{V_{\delta}^{n+1}+V_{\delta}^{n}}{2}\right)+\mathfrak{n}_{\delta}\left(\Phi_{\delta}^{n+\frac{1}{2}}\right) \otimes\left(\frac{V_{\delta}^{n+1}+V_{\delta}^{n}}{2}\right)+I_{h}^{\circ}\left[\frac{f\left(t_{n+1}, \cdot\right)+f\left(t_{n}, \cdot\right)}{2}\right] .
$$

Remark 4.1. Let $A_{\delta}^{\frac{1}{4}}:=\frac{1}{2}\left(V_{\delta}^{\frac{1}{2}}+V_{\delta}^{0}\right)$ and $A_{\delta}^{n+\frac{1}{2}}:=\frac{1}{2}\left(V_{\delta}^{n+1}+V_{\delta}^{n}\right)$ for $n=0, \ldots, N-1$. Then, $(4.3),(4.5)$ and (4.7) are, respectively, equivalent to

$$
2 A_{\delta}^{\frac{1}{4}}-\frac{\tau}{2} \Delta_{h} A_{\delta}^{\frac{1}{4}}-\frac{\tau}{2} g\left(u^{0}\right) \otimes A_{\delta}^{\frac{1}{4}}=2 V_{\delta}^{0}+\frac{\tau}{2} \mathrm{l}_{h}^{\circ}\left[\frac{f\left(t^{\frac{1}{2}}, \cdot\right)+f\left(t_{0}, \cdot\right)}{2}\right]
$$

and

$$
2 A_{\delta}^{n+\frac{1}{2}}-\tau \Delta_{h} A_{\delta}^{n+\frac{1}{2}}-\tau \mathfrak{n}_{\delta}\left(\Phi_{\delta}^{n+\frac{1}{2}}\right) \otimes A_{\delta}^{n+\frac{1}{2}}=2 V_{\delta}^{n}+\tau \mathrm{l}_{h}^{\circ}\left[\frac{f\left(t_{n+1}, \cdot\right)+f\left(t_{n}, \cdot\right)}{2}\right], \quad n=0, \ldots, N-1 .
$$

\subsection{Existence and uniqueness of the (MBRFD) approximations}

Proposition 4.2. Let $g_{\max }^{0}:=\max _{\mathcal{I}}\left|g \circ u_{0}\right|, \delta \geq g_{\max }^{0}$ and $C_{\delta}^{\mathrm{BR}, 1}:=\frac{1}{4} \max _{\mathbb{R}}\left|\mathfrak{n}_{\delta}\right|$. When $\tau \mathrm{C}_{\delta}^{\mathrm{BR}, 1} \leq \frac{1}{2}$, then the (BRFD) approximations are well-defined.

Proof. Let $\zeta \in \mathrm{X}_{h}, \varepsilon \in(0,1]$ and $\mathrm{T}[\varepsilon, \zeta]: \mathrm{X}_{h}^{\circ} \rightarrow \mathrm{X}_{h}^{\circ}$ be a linear operator given by

$$
\mathrm{T}[\varepsilon, \zeta] v:=2 v-\varepsilon \tau \Delta_{h} v-\varepsilon \tau\left[\mathfrak{n}_{\delta}(\zeta) \otimes v\right] \quad \forall v \in \mathbf{X}_{h}^{\circ} .
$$

Since $\delta \geq g_{\max }^{0}$, the definition of $\mathfrak{n}_{\delta}$ yields that $\mathfrak{n}_{\delta}\left(g\left(u^{0}\right)\right)=g\left(u^{0}\right)$. Thus, from (4.9) and (4.8) it is easily seen that the well-posedness of $V_{\delta}^{\frac{1}{2}}$ and $\left(V_{\delta}^{n}\right)_{n=1}^{N}$ follows easily by securing that $\mathrm{T}[\varepsilon, \zeta]$ is invertible. In particular, (4.9) corresponds to the choice $(\varepsilon, \zeta)=\left(1, \Phi^{n+\frac{1}{2}}\right)$ and $(4.8)$ to the choice $(\varepsilon, \zeta)=\left(\frac{1}{2}, g\left(u^{0}\right)\right)$.

Assuming that $\tau \mathrm{C}_{\delta}^{\star} \leq \frac{1}{2}$ and using $(2.5)$ we obtain

$$
\begin{aligned}
(\mathrm{T}[\varepsilon, \zeta] v, v)_{0, h} & =2\|v\|_{0, h}^{2}+\tau \varepsilon|v|_{1, h}^{2}-\tau \varepsilon\left(\mathfrak{n}_{\delta}(\zeta) \otimes v, v\right)_{0, h} \\
& \geq 2\|v\|_{0, h}^{2}+\tau \varepsilon|v|_{0, h}^{2}-\tau \varepsilon\|v\|_{0, h}^{2}\left|\mathfrak{n}_{\delta}(\zeta)\right|_{\infty, h} \\
& \geq \tau \varepsilon|v|_{0, h}^{2}+4\|v\|_{0, h}^{2}\left(\frac{1}{2}-\frac{\tau}{4} \max _{\mathbb{R}}\left|\mathfrak{n}_{\delta}\right|\right) \\
& \geq \tau \varepsilon|v|_{1, h}^{2}+4\|v\|_{0, h}^{2}\left(\frac{1}{2}-\tau C_{\delta}^{\mathrm{BR}, 1}\right) \\
& \geq \tau \varepsilon|v|_{1, h}^{2} \quad \forall v \in \mathrm{X}_{h}^{\circ} .
\end{aligned}
$$

When $v \in \operatorname{Ker}(\mathbf{T}[\varepsilon, \zeta])$, then $(\mathbf{T}[\varepsilon, \zeta] v, v)_{0, h}=0$, which, along with (4.10) yields $|v|_{1, h}=0$ and thus $v=0$. The latter argument shows that $\operatorname{Ker}(\mathrm{T}[\varepsilon, \zeta])=\{0\}$ and, thus, $\mathrm{T}[\varepsilon, \zeta]$ is invertible, since $\mathrm{X}_{h}^{\circ}$ has finite dimension.

Remark 4.3. Let us assume that $\tau C_{\delta}^{\mathrm{BR}, 1} \leq \frac{1}{2}$ and $\delta \geq g_{\max }^{0}$. Since $V_{\delta}^{0}:=u^{0}$ and $V_{\delta}^{\frac{1}{2}}$ is well-defined, in view of (4.3) and (1.6), we conclude that $U^{\frac{1}{2}}$ is, also, well-defined and $U^{\frac{1}{2}}=V_{\delta}^{\frac{1}{2}}$. 


\subsection{Convergence of the (MBRFD) scheme}

First, we establish convergence of the (MBRFD) approximations.

Theorem 4.4. Let $g \in C^{4}(\mathbb{R}), u \in C_{t, x}^{3,0}(Q)$, $\partial_{t}^{\ell} u \in C_{t, x}^{0,1}(Q)$ for $\ell=1,2,3$, and $\partial_{t} u \in C_{t, x}^{0,4}(Q)$. Also, let $u_{\max }:=$ $\max _{Q}|u|, g_{\max }:=\max _{Q}|g \circ u|, g_{\max }^{0}:=\max _{\mathcal{I}}\left|g \circ u_{0}\right|, \delta_{\star} \geq \max \left\{u_{\max }, g_{\max }\right\}$ and $\tau C_{\delta_{\star}}^{\mathrm{BR}, 1} \leq \frac{1}{2}$, where $\mathrm{C}_{\delta_{\star}}^{\mathrm{BR}, \mathrm{l}}:=\frac{1}{4} \max _{\mathbb{R}}\left|\mathfrak{n}_{\delta}\right|$. Then, there exist constants $\mathrm{C}_{\delta_{\star}}^{\mathrm{BCV}, 1} \geq{ }_{\delta_{\star}}^{\mathrm{B}, 1}, \mathrm{C}_{\delta_{\star}}^{\mathrm{BCV}, 2}>0, \mathrm{C}_{\delta_{\star}}^{\mathrm{BC}, 3}>0$ and $\mathrm{C}_{\delta_{\star}}^{\mathrm{BCV}, 4}>0$, independent of $\tau$ and $h$, such that: if $\tau \mathrm{C}_{\delta_{\star}}^{\mathrm{BCV}, 1} \leq \frac{1}{2}$, then

$$
\begin{aligned}
&\left|u^{\frac{1}{2}}-V_{\delta_{\star}}^{\frac{1}{2}}\right|_{1, h} \leq \mathrm{C}_{\delta_{\star}}^{\mathrm{BCV}, 2}\left(\tau^{2}+\tau^{\frac{1}{2}} h^{2}\right), \\
& \max _{0 \leq m \leq N-1}\left\|g\left(u^{m+\frac{1}{2}}\right)-\Phi_{\delta_{\star}}^{m+\frac{1}{2}}\right\|_{0, h}+\max _{0 \leq m \leq N}\left|u^{m}-V_{\delta_{\star}}^{m}\right|_{1, h} \leq \mathrm{C}_{\delta_{\star}}^{\mathrm{BCV}, 3}\left(\tau^{2}+h^{2}\right)
\end{aligned}
$$

and

$$
\max _{0 \leq m \leq N-1}\left|g\left(u^{m+\frac{1}{2}}\right)-\Phi_{\delta_{\star}}^{m+\frac{1}{2}}\right|_{1, h}+\max _{0 \leq m \leq N}\left|u^{m}-V_{\delta_{\star}}^{m}\right|_{2, h} \leq C_{\delta_{\star}}^{\mathrm{BCV}, 4}\left(\tau^{2}+h^{2}\right) .
$$

Proof. To simplify the notation, we set $e^{\frac{1}{2}}:=u^{\frac{1}{2}}-V_{\delta_{\star}}^{\frac{1}{2}} \in \mathrm{X}_{h}^{\circ}, e^{m}:=u^{m}-V_{\delta_{\star}}^{m} \in \mathrm{X}_{h}^{\circ}$ for $m=0, \ldots, N$, and $e_{\text {mid }}^{m}:=g\left(u^{m+\frac{1}{2}}\right)-\Phi_{\delta_{\star}}^{m+\frac{1}{2}} \in \mathrm{X}_{h}^{\circ}$ for $m=0, \ldots, N-1$. In the sequel, we will use the symbol $C$ to denote a generic constant that is independent of $\tau, h$ and $\delta_{\star}$, and may changes value from one line to the other. Also, we will use the symbol $C_{\delta_{\star}}$ to denote a generic constant that depends on $\delta_{\star}$ but is independent of $\tau, h$, and may changes value from one line to the other. We note that the constants $C$ and $C_{\delta_{\star}}$ may depend on the solution $u$ and its partial derivatives.

Part 1: Since $e^{0}=0$, after subtracting (4.3) from (3.12) we obtain

$$
e^{\frac{1}{2}}=\frac{\tau}{4} \Delta_{h} e^{\frac{1}{2}}+\frac{\tau}{4} g\left(u^{0}\right) \otimes e^{\frac{1}{2}}+\frac{\tau}{2} \mathbf{s}^{\frac{1}{4}} .
$$

Next, take the $(\cdot, \cdot)_{0, h}$-inner product of (4.14) with $e^{\frac{1}{2}}$, and then use (2.5), the Cauchy-Schwarz inequality, (3.4), (3.8), (3.16) and the arithmetic mean inequality to get

$$
\begin{aligned}
\left\|e^{\frac{1}{2}}\right\|_{0, h}^{2}+\frac{\tau}{4}\left|e^{\frac{1}{2}}\right|_{1, h}^{2} & =\frac{\tau}{4}\left(g\left(u^{0}\right) \otimes e^{\frac{1}{2}}, e^{\frac{1}{2}}\right)_{0, h}+\frac{\tau}{2}\left(\mathrm{~s}^{\frac{1}{4}}, e^{\frac{1}{2}}\right)_{0, h} \\
& \leq \frac{\tau}{4} g_{\max }^{0}\left\|e^{\frac{1}{2}}\right\|_{0, h}^{2}+\frac{\tau}{2}\left[\left\|\mathbf{s}^{\frac{1}{4}}-\mathrm{r}^{\frac{1}{4}}\right\|_{0, h}+\left\|\mathrm{r}^{\frac{1}{4}}\right\|_{0, h}\right]\left\|e^{\frac{1}{2}}\right\|_{0, h} \\
& \leq \frac{\tau}{4} g_{\max }^{0}\left\|e^{\frac{1}{2}}\right\|_{0, h}^{2}+C\left(\tau^{2}+\tau h^{2}\right)\left\|e^{\frac{1}{2}}\right\|_{0, h} \\
& \leq \frac{\tau}{4} g_{\max }^{0}\left\|e^{\frac{1}{2}}\right\|_{0, h}^{2}+C\left(\tau^{2}+\tau h^{2}\right)^{2}+\frac{1}{2}\left\|e^{\frac{1}{2}}\right\|_{0, h}^{2} .
\end{aligned}
$$

Let $\mathrm{C}_{\delta_{\star}, \mathrm{BR}}^{\mathrm{BR}}:=\max \left\{\frac{1}{2} g_{\max }^{0}, \mathrm{C}_{\delta_{\star}}^{\mathrm{BR}, \mathrm{I}}\right\}$ and $\tau \mathrm{C}_{\delta_{\star}}^{\mathrm{BR}, \mathrm{II}} \leq \frac{1}{2}$. Then, the inequality above yields that

$$
\left\|e^{\frac{1}{2}}\right\|_{0, h}^{2}+\tau\left|e^{\frac{1}{2}}\right|_{1, h}^{2} \leq C\left(\tau^{2}+\tau h^{2}\right)^{2}
$$

Taking the $(\cdot, \cdot)_{0, h}$-inner product of $(4.14)$ with $\Delta_{h} e^{\frac{1}{2}}$, and then using $(2.5)$, we obtain

$$
4\left|e^{\frac{1}{2}}\right|_{1, h}^{2}+\tau\left\|\Delta_{h} e^{\frac{1}{2}}\right\|_{0, h}^{2}=\mathrm{a}^{1}+\mathrm{a}^{2},
$$


where $\mathrm{a}^{1}:=-\tau\left(g\left(u^{0}\right) \otimes e^{\frac{1}{2}}, \Delta_{h} e^{\frac{1}{2}}\right)_{0, h}$ and $\mathrm{a}^{2}:=-2 \tau\left(\mathrm{s}^{\frac{1}{4}}, \Delta_{h} e^{\frac{1}{2}}\right)_{0, h}$. Now, we use the Cauchy-Schwarz inequality, the arithmetic mean inequality and (4.15), to have

$$
\begin{aligned}
\mathrm{a}^{1} & \leq \tau g_{\max }^{0}\left\|e^{\frac{1}{2}}\right\|_{0, h}\left\|\Delta_{h} e^{\frac{1}{2}}\right\|_{0, h} \\
& \leq \tau\left[\frac{3\left(g_{\max }^{0}\right)^{2}}{2}\left\|e^{\frac{1}{2}}\right\|_{0, h}^{2}+\frac{1}{6}\left\|\Delta_{h} e^{\frac{1}{2}}\right\|_{0, h}^{2}\right] \\
& \leq C \tau\left(\tau^{2}+\tau h^{2}\right)^{2}+\frac{\tau}{6}\left\|\Delta_{h} e^{\frac{1}{2}}\right\|_{0, h}^{2} \\
& \leq C\left(\tau^{\frac{5}{2}}+\tau^{\frac{3}{2}} h^{2}\right)^{2}+\frac{\tau}{6}\left\|\Delta_{h} e^{\frac{1}{2}}\right\|_{0, h}^{2} .
\end{aligned}
$$

Also, (3.4), the Cauchy-Schwarz inequality, (3.8) and (3.16) yield

$$
\begin{aligned}
\mathrm{a}^{2} & =-2 \tau\left(\mathrm{s}^{\frac{1}{4}}-\mathrm{r}^{\frac{1}{4}}, \Delta_{h} e^{\frac{1}{2}}\right)_{0, h}-2 \tau\left(\mathrm{r}^{\frac{1}{4}}, \Delta_{h} e^{\frac{1}{2}}\right)_{0, h} \\
& =-2 \tau\left(\mathrm{s}^{\frac{1}{4}}-\mathrm{r}^{\frac{1}{4}}, \Delta_{h} e^{\frac{1}{2}}\right)_{0, h}-2 \tau\left(\mathrm{r}_{A}^{\frac{1}{4}}, \Delta_{h} e^{\frac{1}{2}}\right)_{0, h}+2 \tau\left(\mathrm{r}_{B}^{\frac{1}{4}}, \Delta_{h} e^{\frac{1}{2}}\right)_{0, h} \\
& \leq 2 \tau\left[\left\|\mathrm{s}^{\frac{1}{4}}-\mathrm{r}^{\frac{1}{4}}\right\|_{0, h}+\left\|\mathrm{r}_{A}^{\frac{1}{4}}\right\|_{0, h}\right]\left\|\Delta_{h} e^{\frac{1}{2}}\right\|_{0, h}+2 \tau\left(\mathrm{r}_{B}^{\frac{1}{4}}, \Delta_{h} e^{\frac{1}{2}}\right)_{0, h} \\
& \leq C\left(h^{2}+\tau^{2}\right)\left\|\Delta_{h} e^{\frac{1}{2}}\right\|_{0, h}+2 \tau\left(\mathrm{r}_{B}^{\frac{1}{4}}, \Delta_{h} e^{\frac{1}{2}}\right)_{0, h} .
\end{aligned}
$$

Since $\mathbf{r}_{B}^{\frac{1}{4}} \in \mathrm{X}_{h}^{\circ}$, using (2.4), the Cauchy-Schwarz inequality, (3.9) and the arithmetic mean inequality we arrive at

$$
\begin{aligned}
a^{2} & \leq C \tau\left(\tau^{2}+h^{2}\right)\left\|\Delta_{h} e^{\frac{1}{2}}\right\|_{0, h}-2 \tau\left(\left(\delta_{h} \mathrm{r}_{B}^{\frac{1}{4}}, \delta_{h} e^{\frac{1}{2}}\right)\right)_{0, h} \\
& \leq C \tau\left(\tau^{2}+h^{2}\right)\left\|\Delta_{h} e^{\frac{1}{2}}\right\|_{0, h}+2 \tau\left|\mathrm{r}_{B}^{\frac{1}{4}}\right|_{1, h}\left|e^{\frac{1}{2}}\right|_{1, h} \\
& \leq C \tau\left(\tau^{2}+h^{2}\right)\left\|\Delta_{h} e^{\frac{1}{2}}\right\|_{0, h}+C \tau^{2}\left|e^{\frac{1}{2}}\right|_{1, h} \\
& \leq \tau\left[C\left(\tau^{2}+h^{2}\right)^{2}+\frac{1}{6}\left\|\Delta_{h} e^{\frac{1}{2}}\right\|_{0, h}^{2}\right]+\left[C \tau^{4}+\left|e^{\frac{1}{2}}\right|_{1, h}^{2}\right] \\
& \leq C\left[\left(\tau^{\frac{5}{2}}+\tau^{\frac{1}{2}} h^{2}\right)^{2}+\tau^{4}\right]+\frac{\tau}{6}\left\|\Delta_{h} e^{\frac{1}{2}}\right\|_{0, h}^{2}+\left|e^{\frac{1}{2}}\right|_{1, h}^{2} \\
& \leq C\left(\tau^{2}+\tau^{\frac{1}{2}} h^{2}\right)^{2}+\frac{\tau}{6}\left\|\Delta_{h} e^{\frac{1}{2}}\right\|_{0, h}^{2}+\left|e^{\frac{1}{2}}\right|_{1, h}^{2}
\end{aligned}
$$

In view of (4.16)-(4.18), we arrive at

$$
\left|e^{\frac{1}{2}}\right|_{1, h}^{2}+\tau\left\|\Delta_{h} e^{\frac{1}{2}}\right\|_{0, h}^{2} \leq C\left(\tau^{2}+\tau^{\frac{1}{2}} h^{2}\right)^{2}
$$

which, obviously, yields (4.11).

Since $\delta_{\star} \geq u_{\max }$, we have $\mathfrak{n}_{\delta_{\star}}\left(u^{\frac{1}{2}}\right)=u^{\frac{1}{2}}$, which we use, along with (4.4), (4.1) and (4.15), to obtain

$$
\begin{aligned}
\left\|e_{\text {mid }}^{0}\right\|_{0, h}^{2} & =\left\|g\left(\mathfrak{n}_{\delta_{\star}}\left(u^{\frac{1}{2}}\right)\right)-g\left(\mathfrak{n}_{\delta_{\star}}\left(V_{\delta_{\star}}^{\frac{1}{2}}\right)\right)\right\|_{0, h}^{2} \\
& \leq \sup _{\mathbb{R}}\left|\left(g \circ \mathfrak{n}_{\delta_{\star}}\right)^{\prime}\right|^{2}\left\|e^{\frac{1}{2}}\right\|_{0, h}^{2} \\
& \leq C_{\delta_{\star}}\left(\tau^{2}+\tau h^{2}\right)^{2}
\end{aligned}
$$


Also, using (2.7) (with $\left.\mathfrak{g}=g \circ \mathfrak{n}_{\delta_{\star}}\right),(2.3)$ and (4.19), we get

$$
\begin{aligned}
\left|e_{\text {mid }}^{0}\right|_{1, h}^{2} & =\left|g\left(\mathfrak{n}_{\delta_{\star}}\left(u^{\frac{1}{2}}\right)\right)-g\left(\mathfrak{n}_{\delta_{\star}}\left(V_{\delta_{\star}}^{\frac{1}{2}}\right)\right)\right|_{1, h}^{2} \\
& \leq 2 \sup _{\mathbb{R}}\left|\left(g \circ \mathfrak{n}_{\delta_{\star}}\right)^{\prime}\right|^{2}\left|e^{\frac{1}{2}}\right|_{1, h}^{2}+2 \sup _{\mathbb{R}}\left|\left(g \circ \mathfrak{n}_{\delta_{\star}}\right)^{\prime \prime}\right|^{2}\left\|\delta_{h} g\left(u^{\frac{1}{2}}\right)\right\|_{\infty, h}^{2}\left\|e^{\frac{1}{2}}\right\|_{0, h}^{2} \\
& \leq C_{\delta_{\star}}\left|e^{\frac{1}{2}}\right|_{1, h}^{2} \\
& \leq C_{\delta_{\star}}\left(\tau^{2}+\tau^{\frac{1}{2}} h^{2}\right)^{2} .
\end{aligned}
$$

Part 2: We subtract (4.5) and (4.7) from (3.13), to obtain the following error equations:

$$
2\left(e^{n+1}-e^{n}\right)=\tau \Delta_{h}\left(e^{n+1}+e^{n}\right)+\sum_{\kappa=1}^{3} \mathrm{Q}^{\kappa, n}, \quad n=0, \ldots, N-1,
$$

where $\mathrm{Q}^{1, n}:=2 \tau \mathrm{s}^{n+\frac{1}{2}}, \mathrm{Q}^{2, n}:=\tau \mathfrak{n}_{\delta_{\star}}\left(\Phi_{\delta_{\star}}^{n+\frac{1}{2}}\right) \otimes\left(e^{n+1}+e^{n}\right)$ and

$$
\mathrm{Q}^{3, n}:=\tau\left[g\left(u^{n+\frac{1}{2}}\right)-\mathfrak{n}_{\delta_{\star}}\left(\Phi_{\delta_{\star}}^{n+\frac{1}{2}}\right)\right] \otimes\left(u^{n+1}+u^{n}\right) .
$$

Taking the inner product $(\cdot, \cdot)_{0, h}$ of $(4.22)$ with $\left(e^{n+1}-e^{n}\right)$, and then, use $(2.4)$, we arrive at

$$
2\left\|e^{n+1}-e^{n}\right\|_{0, h}^{2}+\tau\left[\left|e^{n+1}\right|_{1, h}^{2}-\left|e^{n}\right|_{1, h}^{2}\right]=\sum_{\kappa=1}^{3}\left(\mathrm{Q}^{\kappa, n}, e^{n+1}-e^{n}\right)_{0, h}, \quad n=0, \ldots, N-1 .
$$

Let $n \in\{0 \ldots, N-1\}$. Using the Cauchy-Schwarz inequality, the arithmetic mean inequality, (3.8) and (3.16), we have

$$
\begin{aligned}
\left(\mathrm{Q}^{1, n}, e^{n+1}-e^{n}\right)_{0, h} & \leq 2 \tau\left[\left\|\mathrm{s}^{n+\frac{1}{2}}-\mathrm{r}^{n+\frac{1}{2}}\right\|_{0, h}+\left\|\mathrm{r}^{n+\frac{1}{2}}\right\|_{0, h}\right]\left\|e^{n+1}-e^{n}\right\|_{0, h} \\
& \leq C \tau\left(\tau^{2}+h^{2}\right)\left\|e^{n+1}-e^{n}\right\|_{0, h} \\
& \leq C \tau^{2}\left(\tau^{2}+h^{2}\right)^{2}+\frac{1}{6}\left\|e^{n+1}-e^{n}\right\|_{0, h}^{2} .
\end{aligned}
$$

Next, we use the Cauchy-Schwarz inequality, (2.3), (4.1) and the arithmetic mean inequality, to get

$$
\begin{aligned}
\left(\mathrm{Q}^{2, n}, e^{n+1}-e^{n}\right)_{0, h} & \leq \tau\left|\mathfrak{n}_{\delta_{\star}}\left(\Phi_{\delta_{\star}}^{n+\frac{1}{2}}\right)\right|_{\infty, h}\left\|e^{n+1}+e^{n}\right\|_{0, h}\left\|e^{n+1}-e^{n}\right\|_{0, h} \\
& \leq C_{\delta_{\star}} \tau\left|e^{n+1}+e^{n}\right|_{1, h}\left\|e^{n+1}-e^{n}\right\|_{0, h} \\
& \leq C_{\delta_{\star}} \tau^{2}\left[\left|e^{n+1}\right|_{1, h}^{2}+\left|e^{n}\right|_{1, h}^{2}\right]+\frac{1}{6}\left\|e^{n+1}-e^{n}\right\|_{0, h}^{2} .
\end{aligned}
$$

Finally, taking into account that $\delta_{\star} \geq g_{\max }$, we apply the Cauchy-Schwarz inequality, (4.1) and the arithmetic mean inequality to obtain

$$
\begin{aligned}
\left(\mathrm{Q}^{3, n}, e^{n+1}-e^{n}\right)_{0, h} & \leq 2 \tau u_{\max }\left\|\mathfrak{n}_{\delta_{\star}}\left(g\left(u^{n+\frac{1}{2}}\right)\right)-\mathfrak{n}_{\delta_{\star}}\left(\Phi_{\delta_{\star}}^{n+\frac{1}{2}}\right)\right\|_{0, h}\left\|e^{n+1}-e^{n}\right\|_{0, h} \\
& \leq C \tau \max _{\mathbb{R}}\left|\mathfrak{n}_{\delta_{\star}}^{\prime}\right|\left\|g\left(u^{n+\frac{1}{2}}\right)-\Phi_{\delta_{\star}}^{n+\frac{1}{2}}\right\|_{0, h}\left\|e^{n+1}-e^{n}\right\|_{0, h} \\
& \leq C_{\delta_{\star}} \tau\left\|e_{\text {mid }}^{n}\right\|_{0, h}\left\|e^{n+1}-e^{n}\right\|_{0, h} \\
& \leq C_{\delta_{\star}} \tau^{2}\left\|e_{\text {mid }}^{n}\right\|_{0, h}^{2}+\frac{1}{6}\left\|e^{n+1}-e^{n}\right\|_{0, h}^{2} .
\end{aligned}
$$

From (4.23)-(4.26), we conclude that there exists a constant $\mathrm{C}_{\delta_{\star}}^{\mathrm{BR}, \mathrm{III}}>0$, such that

$$
\begin{aligned}
\left\|e^{n+1}-e^{n}\right\|_{0, h}^{2}+\tau\left|e^{n+1}\right|_{1, h}^{2} \leq & \tau\left|e^{n}\right|_{1, h}^{2}+\mathrm{C}_{\delta_{\star}}^{\mathrm{BR}, \mathrm{II}} \tau^{2}\left[\left|e^{n+1}\right|_{1, h}^{2}+\left|e^{n}\right|_{1, h}^{2}+\left\|e_{\text {mid }}^{n}\right\|_{0, h}^{2}\right] \\
& +C \tau^{2}\left(\tau^{2}+h^{2}\right)^{2}, \quad n=0, \ldots, N-1 .
\end{aligned}
$$


Let us find an error equation governing the midpoint error $e_{\text {mid }}^{n}$. Subtracting (4.6) from (3.1) and using (4.1) and the assumption $\delta_{\star} \geq u_{\max }$, we obtain

$$
e_{\text {mid }}^{n}+e_{\text {mid }}^{n-1}=2\left[g\left(\mathfrak{n}_{\delta_{\star}}\left(u^{n}\right)\right)-g\left(\mathfrak{n}_{\delta_{\star}}\left(V_{\delta_{\star}}^{n}\right)\right)\right]+2 \mathbf{r}^{n}, \quad n=1, \ldots, N-1,
$$

which, easily, yields that

$$
e_{\text {mid }}^{n}-e_{\text {mid }}^{n-2}=2 \sigma^{n}+2\left(\mathrm{r}^{n}-\mathrm{r}^{n-1}\right), \quad n=2, \ldots, N-1,
$$

where $\sigma^{n} \in \mathrm{X}_{h}^{\circ}$ is defined by

$$
\sigma^{n}:=g\left(\mathfrak{n}_{\delta_{\star}}\left(V_{\delta_{\star}}^{n-1}\right)\right)-g\left(\mathfrak{n}_{\delta_{\star}}\left(V_{\delta_{\star}}^{n}\right)\right)-g\left(\mathfrak{n}_{\delta_{\star}}\left(u^{n-1}\right)\right)+g\left(\mathfrak{n}_{\delta_{\star}}\left(u^{n}\right)\right) .
$$

Then, we use (2.8) (with $\left.\mathfrak{g}=g \circ \mathfrak{n}_{\delta_{\star}}\right),(4.1)$ and the mean value theorem, to get

$$
\begin{aligned}
\left\|\sigma^{n}\right\|_{0, h} \leq & \sup _{\mathbb{R}}\left|\left(g \circ \mathfrak{n}_{\delta_{\star}}\right)^{\prime}\right|\left\|e^{n}-e^{n-1}\right\|_{0, h} \\
& +\sup _{\mathbb{R}}\left|\left(g \circ \mathfrak{n}_{\delta_{\star}}\right)^{\prime \prime}\right|\left|u^{n-1}-u^{n}\right|_{\infty, h}\left[\left\|e^{n}-e^{n-1}\right\|_{0, h}+\left\|e^{n}\right\|_{0, h}\right] \\
& \leq C_{\delta_{\star}}\left[\left\|e^{n}-e^{n-1}\right\|_{0, h}+\tau\left\|e^{n}\right\|_{0, h}\right], \quad n=2, \ldots, N-1 .
\end{aligned}
$$

Taking the $(\cdot, \cdot)_{0, h}$ inner product of both sides of (4.29) with $\tau\left(e_{\text {mid }}^{n}+e_{\text {mid }}^{n-2}\right)$, and then using the Cauchy-Schwarz inequality, (4.31), (3.11) and (2.3), it follows that

$$
\begin{aligned}
\tau\left\|e_{\text {mid }}^{n}\right\|_{0, h}^{2}-\tau\left\|e_{\text {mid }}^{n-2}\right\|_{0, h}^{2} \leq & {\left[2 \tau\left\|\sigma^{n}\right\|_{0, h}+2 \tau\left\|\mathrm{r}^{n}-\mathrm{r}^{n-1}\right\|_{0, h}\right]\left\|e_{\text {mid }}^{n}+e_{\text {mid }}^{n-2}\right\|_{0, h} } \\
\leq & C_{\delta_{\star}}\left[\tau\left\|e^{n}-e^{n-1}\right\|_{0, h}+\tau^{2}\left\|e^{n}\right\|_{0, h}\right]\left\|e_{\text {mid }}^{n}+e_{\text {mid }}^{n-2}\right\|_{0, h} \\
& +C \tau^{4}\left\|e_{\text {mid }}^{n}+e_{\text {mid }}^{n-2}\right\|_{0, h} \\
\leq & C_{\delta_{\star}} \tau\left\|e^{n}-e^{n-1}\right\|_{0, h}\left(\left\|e_{\text {mid }}^{n}\right\|_{0, h}+\left\|e_{\text {mid }}^{n-2}\right\|_{0, h}\right) \\
& +C_{\delta_{\star}} \tau^{2}\left|e^{n}\right|_{1, h}\left(\left\|e_{\text {mid }}^{n}\right\|_{0, h}+\left\|e_{\text {mid }}^{n-2}\right\|_{0, h}\right) \\
& +C \tau^{4}\left(\left\|e_{\text {mid }}^{n}\right\|_{0, h}+\left\|e_{\text {mid }}^{n-2}\right\|_{0, h}\right), \quad n=2, \ldots, N-1 .
\end{aligned}
$$

Now, we apply the arithmetic mean inequality to obtain

$$
\begin{aligned}
\tau\left\|e_{\text {mid }}^{n}\right\|_{0, h}^{2}-\tau\left\|e_{\text {mid }}^{n-2}\right\|_{0, h}^{2} \leq & C_{\delta_{\star}} \tau^{2}\left(\left\|e_{\text {mid }}^{n}\right\|_{0, h}+\left\|e_{\text {mid }}^{n-2}\right\|_{0, h}\right)^{2}+\left\|e^{n}-e^{n-1}\right\|_{0, h}^{2} \\
& +C_{\delta_{\star}} \tau^{2}\left[\left|e^{n}\right|_{1, h}^{2}+\left(\left\|e_{\text {mid }}^{n}\right\|_{0, h}+\left\|e_{\text {mid }}^{n-2}\right\|_{0, h}\right)^{2}\right] \\
& +C \tau^{6}+\tau^{2}\left(\left\|e_{\text {mid }}^{n}\right\|_{0, h}+\left\|e_{\text {mid }}^{n-2}\right\|_{0, h}\right)^{2} \\
\leq & C_{\delta_{\star}} \tau^{2}\left(\left\|e_{\text {mid }}^{n}\right\|_{0, h}^{2}+\left\|e_{\text {mid }}^{n-2}\right\|_{0, h}^{2}\right)+\left\|e^{n}-e^{n-1}\right\|_{0, h}^{2} \\
& +C_{\delta_{\star}} \tau^{2}\left(\left|e^{n}\right|_{1, h}^{2}+\left\|e_{\text {mid }}^{n}\right\|_{0, h}^{2}+\left\|e_{\text {mid }}^{n-2}\right\|_{0, h}^{2}\right) \\
& +C \tau^{6}+2 \tau^{2}\left(\left\|e_{\text {mid }}^{n}\right\|_{0, h}^{2}+\left\|e_{\text {mid }}^{n-2}\right\|_{0, h}^{2}\right), \quad n=2, \ldots, N-1 .
\end{aligned}
$$

Thus, we arrive at

$$
\begin{aligned}
\tau\left\|e_{\text {mid }}^{n}\right\|_{0, h}^{2}+\tau\left\|e_{\text {mid }}^{n-1}\right\|_{0, h}^{2} \leq & \tau\left\|e_{\text {mid }}^{n-1}\right\|_{0, h}^{2}+\tau\left\|e_{\text {mid }}^{n-2}\right\|_{0, h}^{2}+\left\|e^{n}-e^{n-1}\right\|_{0, h}^{2}+C \tau^{6} \\
& +C_{\delta_{\star}} \tau^{2}\left[\left|e^{n}\right|_{1, h}^{2}+\left\|e_{\text {mid }}^{n}\right\|_{0, h}^{2}+\left\|e_{\text {mid }}^{n-2}\right\|_{0, h}^{2}\right], \quad n=2, \ldots, N-1 .
\end{aligned}
$$

From (4.27) and (4.32), we conclude that there exists a constant $\mathrm{C}_{\delta_{\star}}^{\mathrm{BR}, \mathrm{IV}}>0$ such that:

$$
\left(1-\mathrm{C}_{\delta_{\star}, \mathrm{BR}}^{\mathrm{BR}} \tau\right) \mathrm{E}^{n+1} \leq\left(1+\mathrm{C}_{\delta_{\star}}^{\mathrm{BR}, \mathrm{V}} \tau\right) \mathrm{E}^{n}+C \tau^{2}\left(\tau^{2}+h^{2}\right)^{2}, \quad n=2, \ldots, N-1,
$$


where

$$
\mathrm{E}^{m}:=\left\|e^{m}-e^{m-1}\right\|_{0, h}^{2}+\tau\left(\left|e^{m}\right|_{1, h}^{2}+\left\|e_{\text {mid }}^{m-1}\right\|_{0, h}^{2}+\left\|e_{\text {mid }}^{m-2}\right\|_{0, h}^{2}\right), \quad n=2, \ldots, N .
$$

Assuming that $\tau \mathrm{C}_{\delta_{\star}}^{\mathrm{BR}, \mathrm{V}} \leq \frac{1}{2}$ with $\mathrm{C}_{\delta_{\star}}^{\mathrm{BR}, \mathrm{V}}:=\max \left\{\mathrm{C}_{\delta}^{\mathrm{BR}, \mathrm{III}}, \mathrm{C}_{\delta_{\star}, \mathrm{V}}^{\mathrm{BR}}\right\}$, and applying a standard discrete Gronwall argument, from (4.33) we arrive at

$$
\max _{2 \leq m \leq N} \mathrm{E}^{m} \leq C_{\delta_{\star}}\left[\mathrm{E}^{2}+\tau\left(\tau^{2}+h^{2}\right)^{2}\right] .
$$

Since $e^{0}=0$, the later inequality along with (4.34) obviously yield that

$$
\begin{aligned}
\tau\left[\max _{0 \leq m \leq N}\left|e^{m}\right|_{1, h}^{2}+\max _{0 \leq m \leq N-1}\left\|e_{\text {mid }}^{m}\right\|_{0, h}^{2}\right] \leq & C_{\delta_{\star}}\left[\left\|e^{2}-e^{1}\right\|_{0, h}^{2}+\tau\left|e^{2}\right|_{1, h}^{2}+\tau\left|e^{1}\right|_{1, h}^{2}\right. \\
& \left.+\tau\left\|e_{\text {mid }}^{1}\right\|_{0, h}^{2}+\tau\left\|e_{\text {mid }}^{0}\right\|_{0, h}^{2}+\tau\left(\tau^{2}+h^{2}\right)^{2}\right] .
\end{aligned}
$$

Setting $n=0$ in (4.27) and using that $e^{0}=0$, we obtain

$$
\tau\left|e^{1}\right|_{1, h}^{2} \leq C_{\delta_{\star}}\left[\tau^{2}\left(\tau^{2}+h^{2}\right)^{2}+\tau^{2}\left\|e_{\text {mid }}^{0}\right\|_{0, h}^{2}\right] .
$$

Now, we set $n=1$ in (4.27) and use (4.36), to have

$$
\begin{aligned}
\left\|e^{2}-e^{1}\right\|_{0, h}^{2}+\tau\left|e^{2}\right|_{1, h}^{2} & \leq C_{\delta_{\star}}\left[\tau^{2}\left(\tau^{2}+h^{2}\right)^{2}+\tau\left|e^{1}\right|_{1, h}^{2}+\tau^{2}\left\|e_{\text {mid }}^{1}\right\|_{0, h}^{2}\right] \\
& \leq C_{\delta_{\star}}\left[\tau^{2}\left(\tau^{2}+h^{2}\right)^{2}+\tau^{2}\left\|e_{\text {mid }}^{0}\right\|_{0, h}^{2}+\tau^{2}\left\|e_{\text {mid }}^{1}\right\|_{0, h}^{2}\right] .
\end{aligned}
$$

Also, we set $n=1$ in (4.28) and then use (3.10), (2.3) and (4.36), to get

$$
\begin{aligned}
\left\|e_{\text {mid }}^{1}\right\|_{0, h}^{2} & \leq\left[\left\|e_{\text {mid }}^{0}\right\|_{0, h}+2 \sup _{\mathbb{R}}\left|\left(g \circ \mathfrak{n}_{\delta_{\star}}\right)^{\prime}\right|\left\|e^{1}\right\|_{0, h}+2\left\|\mathrm{r}^{1}\right\|_{0, h}\right]^{2} \\
& \leq C_{\delta_{\star}}\left(\left\|e_{\text {mid }}^{0}\right\|_{0, h}^{2}+\left\|e^{1}\right\|_{0, h}^{2}+\tau^{4}\right) \\
& \leq C_{\delta_{\star}}\left(\left\|e_{\text {mid }}^{0}\right\|_{0, h}^{2}+\left|e^{1}\right|_{1, h}^{2}+\tau^{4}\right) \\
& \leq C_{\delta_{\star}}\left[\left\|e_{\text {mid }}^{0}\right\|_{0, h}^{2}+\left(\tau^{2}+h^{2}\right)^{2}\right] .
\end{aligned}
$$

From (4.35) to (4.38), we arrive at

$$
\max _{0 \leq m \leq N}\left|e^{m}\right|_{1, h}^{2}+\max _{0 \leq m \leq N-1}\left\|e_{\text {mid }}^{m}\right\|_{0, h}^{2} \leq C_{\delta_{\star}}\left[\left(\tau^{2}+h^{2}\right)^{2}+\left\|e_{\text {mid }}^{0}\right\|_{0, h}^{2}\right] .
$$

Thus, (4.12) follows, easily, from (4.39) and (4.20).

Part 3: Let us define $\rho^{m}:=\mathrm{R}_{h}\left[u\left(t^{m}, \cdot\right)\right]-u^{m} \in \mathrm{X}_{h}^{\circ}$ and $\eta^{m}:=V_{\delta_{\star}}^{m}-\mathrm{R}_{h}\left[u\left(t^{m}, \cdot\right)\right] \in \mathrm{X}_{h}^{\circ}$ for $m=0, \ldots, N$. Then, using (4.5), (4.7), (3.3) and (2.1) we get

$$
2\left(\eta^{n+1}-\eta^{n}\right)=\tau \Delta_{h}\left(\eta^{n+1}+\eta^{n}\right)+\sum_{\kappa=1}^{4} \mathrm{~B}^{\kappa, n}, \quad n=0, \ldots, N-1,
$$

where

$$
\begin{aligned}
& \mathrm{B}^{1, n}:=2 \tau\left(\frac{u^{n+1}-u^{n}}{\tau}-\mathrm{R}_{h}\left[\frac{u\left(t^{n+1}, \cdot\right)-u\left(t^{n}, \cdot\right)}{\tau}\right]\right), \\
& \mathrm{B}^{2, n}:=-2 \tau \mathrm{r}^{n+\frac{1}{2}} \\
& \mathrm{~B}^{3, n}:=-\tau \mathfrak{n}_{\delta_{\star}}\left(\Phi_{\delta_{\star}}^{n+\frac{1}{2}}\right) \otimes\left(e^{n+1}+e^{n}\right), \\
& \mathrm{B}^{4, n}:=\tau\left[\mathfrak{n}_{\delta_{\star}}\left(\Phi_{\delta_{\star}}^{n+\frac{1}{2}}\right)-\mathfrak{n}_{\delta_{\star}}\left(g\left(u^{n+\frac{1}{2}}\right)\right)\right] \otimes\left(u^{n+1}+u^{n}\right) .
\end{aligned}
$$


Take the $(\cdot, \cdot)_{0, h}$-inner product of (4.40) with $\Delta_{h}\left(\eta^{n+1}-\eta^{n}\right)$, and then, use (2.5) and (2.4), to have

$$
2\left|\eta^{n+1}-\eta^{n}\right|_{1, h}^{2}+\tau\left[\left\|\Delta_{h} \eta^{n+1}\right\|_{0, h}^{2}-\left\|\Delta_{h} \eta^{n}\right\|_{0, h}^{2}\right]=\sum_{\kappa=1}^{4}\left(\left(\delta_{h} \mathrm{~B}^{\kappa, n}, \delta_{h}\left(\eta^{n+1}-\eta^{n}\right)\right)\right)_{0, h}
$$

for $n=0, \ldots, N-1$.

Let $n \in\{0 \ldots, N-1\}$. Using the Cauchy-Schwarz inequality, the arithmetic mean inequality, (3.9) and (3.21), we have

and

$$
\begin{aligned}
\left(\left(\delta_{h} \mathrm{~B}^{1, n}, \delta_{h}\left(\eta^{n+1}-\eta^{n}\right)\right)\right)_{0, h} & \leq\left|\mathrm{B}^{1, n}\right|_{1, h}\left|\eta^{n+1}-\eta^{n}\right|_{1, h} \\
& \leq C \tau h^{2}\left|\eta^{n+1}-\eta^{n}\right|_{1, h} \\
& \leq C \tau^{2} h^{4}+\frac{1}{6}\left|\eta^{n+1}-\eta^{n}\right|_{1, h}^{2}
\end{aligned}
$$

$$
\begin{aligned}
\left(\left(\delta_{h} \mathrm{~B}^{2, n}, \delta_{h}\left(\eta^{n+1}-\eta^{n}\right)\right)\right)_{0, h} & \leq 2 \tau\left|\mathrm{r}^{n+\frac{1}{2}}\right|_{1, h}\left|\eta^{n+1}-\eta^{n}\right|_{1, h} \\
& \leq C \tau^{3}\left|\eta^{n+1}-\eta^{n}\right|_{1, h} \\
& \leq C \tau^{6}+\frac{1}{6}\left|\eta^{n+1}-\eta^{n}\right|_{1, h}^{2}
\end{aligned}
$$

Using, again, the Cauchy-Schwarz inequality and the arithmetic mean inequality, we get

$$
\left(\left(\delta_{h}\left(\mathrm{~B}^{3, n}+\mathrm{B}^{4, n}\right), \delta_{h}\left(\eta^{n+1}-\eta^{n}\right)\right)\right)_{0, h} \leq \frac{3}{2} \tau^{2}\left(\left|\mathrm{c}^{3, n}\right|_{1, h}^{2}+\left|\mathrm{c}^{4, n}\right|_{1, h}^{2}\right)+\frac{2}{6}\left|\eta^{n+1}-\eta^{n}\right|_{1, h}^{2}
$$

where

$$
\begin{aligned}
c^{3, n} & :=\mathfrak{n}_{\delta_{\star}}\left(\Phi_{\delta_{\star}}^{n+\frac{1}{2}}\right) \otimes\left(e^{n+1}+e^{n}\right), \\
c^{4, n} & :=\left(g\left(u^{n+\frac{1}{2}}\right)-\mathfrak{n}_{\delta_{\star}}\left(\Phi_{\delta_{\star}}^{n+\frac{1}{2}}\right)\right) \otimes\left(u^{n+1}+u^{n}\right) .
\end{aligned}
$$

Using (4.1), (2.2), (4.12), (3.20) and (2.6), we get

$$
\begin{aligned}
\left|c^{3, n}\right|_{1, h}^{2} & \leq\left[\left|\mathfrak{n}_{\delta_{\star}}\left(\Phi_{\delta_{\star}}^{n+\frac{1}{2}}\right)\right|_{1, h}\left|e^{n+1}+e^{n}\right|_{\infty, h}+\left|\mathfrak{n}_{\delta_{\star}}\left(\Phi_{\delta_{\star}}^{n+\frac{1}{2}}\right)\right|_{\infty, h}\left|e^{n+1}+e^{n}\right|_{1, h}\right]^{2} \\
& \leq C\left[\left|\mathfrak{n}_{\delta_{\star}}\left(\Phi_{\delta_{\star}}^{n+\frac{1}{2}}\right)\right|_{1, h}+\left|\mathfrak{n}_{\delta_{\star}}\left(\Phi_{\delta_{\star}}^{n+\frac{1}{2}}\right)\right|_{\infty, h}\right]^{2}\left|e^{n+1}+e^{n}\right|_{1, h}^{2} \\
& \leq C\left[\sup _{\mathbb{R}}\left|\mathfrak{n}_{\delta_{\star}}^{\prime}\right|\left|\Phi_{\delta_{\star}}^{n+\frac{1}{2}}\right|_{1, h}+\sup _{\mathbb{R}}\left|\mathfrak{n}_{\delta_{\star}}\right|\right]^{2}\left(\left|e^{n+1}\right|_{1, h}^{2}+\left|e^{n}\right|_{1, h}^{2}\right) \\
& \leq C_{\delta_{\star}}\left[1+\left|\Phi_{\delta_{\star}}^{n+\frac{1}{2}}-g\left(u^{n+\frac{1}{2}}\right)\right|_{1, h}+\left|g\left(u^{n+\frac{1}{2}}\right)\right|_{1, h}\right]^{2}\left(\left|e^{n+1}\right|_{1, h}^{2}+\left|e^{n}\right|_{1, h}^{2}\right) \\
& \leq C_{\delta_{\star}}\left(1+\left|e_{\text {mid }}^{n}\right|_{1, h}^{2}\right)\left(\left|e^{n+1}\right|_{1, h}^{2}+\left|e^{n}\right|_{1, h}^{2}\right) \\
& \leq C_{\delta_{\star}}\left[\left|e_{\text {mid }}^{n}\right|_{1, h}^{2}+\left|e^{n+1}\right|_{1, h}^{2}+\left|e^{n}\right|_{1, h}^{2}\right] \\
& \leq C_{\delta_{\star}}\left[\left|e_{\text {mid }}^{n}\right|_{1, h}^{2}+\left|\rho^{n+1}\right|_{1, h}^{2}+\left|\rho^{n}\right|_{1, h}^{2}+\left|\eta^{n+1}\right|_{1, h}^{2}+\left|\eta^{n}\right|_{1, h}^{2}\right] \\
& \leq C_{\delta_{\star}}\left[\left|e_{\text {mid }}^{n}\right|_{1, h}^{2}+h^{4}+\left|\eta^{n+1}\right|_{2, h}^{2}+\left|\eta^{n}\right|_{2, h}^{2}\right] .
\end{aligned}
$$

Also, combining (2.2), (2.3), (2.7) (with $\mathfrak{g}=\mathfrak{n}_{\delta_{\star}}$ ) and the assumption $\delta_{\star}>g_{\max }$ we obtain

$$
\begin{aligned}
\left|c^{4, n}\right|_{1, h}^{2} \leq & C\left[\left|\mathfrak{n}_{\delta_{\star}}\left(g\left(u^{n+\frac{1}{2}}\right)\right)-\mathfrak{n}_{\delta_{\star}}\left(\Phi_{\delta_{\star}}^{n+\frac{1}{2}}\right)\right|_{1, h}\left|u^{n+1}+u^{n}\right|_{\infty, h}\right. \\
& \left.+\left\|\mathfrak{n}_{\delta_{\star}}\left(g\left(u^{n+\frac{1}{2}}\right)\right)-\mathfrak{n}_{\delta_{\star}}\left(\Phi_{\delta_{\star}}^{n+\frac{1}{2}}\right)\right\|_{0, h}\left\|\delta_{h}\left(u^{n+1}+u^{n}\right)\right\| \|_{\infty, h}\right]^{2} \\
\leq & C\left|\mathfrak{n}_{\delta_{\star}}\left(\Phi_{\delta_{\star}}^{n+\frac{1}{2}}\right)-\mathfrak{n}_{\delta_{\star}}\left(g\left(u^{n+\frac{1}{2}}\right)\right)\right|_{1, h}^{2} \\
\leq & C\left[\sup _{\mathbb{R}}\left|\mathfrak{n}_{\delta_{\star}}^{\prime}\right|\left|e_{\text {mid }}^{n}\right|_{1, h}+\max _{\mathbb{R}}\left|\mathfrak{n}_{\delta_{\star}}^{\prime \prime}\right|\left\|\delta_{h}\left(g\left(u^{n+\frac{1}{2}}\right)\right)\right\|\left\|_{\infty, h}\right\| e_{\text {mid }}^{n} \|_{0, h}\right]^{2} \\
\leq & C_{\delta_{\star}}\left|e_{\text {mid }}^{n}\right|_{1, h}^{2} .
\end{aligned}
$$


Thus, (4.44)-(4.46) yield

$$
\begin{aligned}
\left(\left(\delta_{h}\left(\mathrm{~B}^{3, n}+\mathrm{B}^{4, n}\right), \delta_{h}\left(\eta^{n+1}-\eta^{n}\right)\right)\right)_{0, h} \leq & C_{\delta_{\star}} \tau^{2}\left[h^{4}+\left|e_{\text {mid }}^{n}\right|_{1, h}^{2}+\left|\eta^{n+1}\right|_{2, h}^{2}+\left|\eta^{n}\right|_{2, h}^{2}\right] \\
& +\frac{1}{3}\left|\eta^{n+1}-\eta^{n}\right|_{1, h}^{2} .
\end{aligned}
$$

From (4.41) to (4.43) and (4.47), we conclude that there exists a constant $C_{\delta_{\star}}^{\text {BR,VI }}>0$, such that

$$
\begin{aligned}
\left|\eta^{n+1}-\eta^{n}\right|_{1, h}^{2}+\tau\left|\eta^{n+1}\right|_{2, h}^{2} \leq & \tau\left|\eta^{n}\right|_{2, h}^{2}+\mathrm{C}_{\delta_{\star}}^{\mathrm{BR}, \mathrm{V} 1} \tau^{2}\left(\left|e_{\mathrm{mid}}^{n}\right|_{1, h}^{2}+\left|\eta^{n+1}\right|_{2, h}^{2}+\left|\eta^{n}\right|_{2, h}^{2}\right) \\
& +C \tau^{2}\left(\tau^{2}+h^{2}\right)^{2}, \quad n=0, \ldots, N-1 .
\end{aligned}
$$

Taking the $(\cdot, \cdot)_{0, h}$ inner product of both sides of (4.29) by $\tau \Delta_{h}\left(e_{\text {mid }}^{n}+e_{\text {mid }}^{n-2}\right)$, and using (2.4), the CauchySchwarz inequality and (3.11), we have

$$
\begin{aligned}
\tau\left|e_{\text {mid }}^{n}\right|_{1, h}^{2}-\tau\left|e_{\text {mid }}^{n-2}\right|_{1, h}^{2}= & 2 \tau\left(\left(\delta_{h} \sigma^{n}, \delta_{h}\left(e_{\text {mid }}^{n}+e_{\text {mid }}^{n-2}\right)\right)\right)_{0, h} \\
& +2 \tau\left(\left(\delta_{h}\left(\mathrm{r}^{n}-\mathrm{r}^{n-1}\right), \delta_{h}\left(e_{\text {mid }}^{n}+e_{\text {mid }}^{n-2}\right)\right)\right)_{0, h} \\
\leq & 2 \tau\left(\left|\sigma^{n}\right|_{1, h}+\left|\mathrm{r}^{n}-\mathrm{r}^{n-1}\right|_{1, h}\right)\left(\left|e_{\text {mid }}^{n}\right|_{1, h}+\left|e_{\text {mid }}^{n-2}\right|_{1, h}\right) \\
\leq & 2 \tau\left(\left|\sigma^{n}\right|_{1, h}+\tau^{3}\right)\left(\left|e_{\text {mid }}^{n}\right|_{1, h}+\left|e_{\text {mid }}^{n-2}\right|_{1, h}\right), \quad n=2, \ldots, N-1 .
\end{aligned}
$$

Using (4.30), (2.9) (with $\left.\mathfrak{g}=g \circ \mathfrak{n}_{\delta_{\star}}\right),(2.3),(4.12)$ and (3.21), we get

$$
\begin{aligned}
\left|\sigma^{n}\right|_{1, h} & \leq C_{\delta_{\star}}\left(\left|e^{n}-e^{n-1}\right|_{1, h}+\tau\left|e^{n}\right|_{1, h}\right) \\
& \leq C_{\delta_{\star}}\left[\left|\eta^{n}-\eta^{n-1}\right|_{1, h}+\left|\rho^{n}-\rho^{n-1}\right|_{1, h}+\tau\left(\tau^{2}+h^{2}\right)\right] \\
& \leq C_{\delta_{\star}}\left[\left|\eta^{n}-\eta^{n-1}\right|_{1, h}+\tau\left(\tau^{2}+h^{2}\right)\right], \quad n=2, \ldots, N-1 .
\end{aligned}
$$

Then, (4.49), (4.50) and the arithmetic mean inequality, yield

$$
\begin{aligned}
\tau\left|e_{\text {mid }}^{n}\right|_{1, h}^{2}+\tau\left|e_{\text {mid }}^{n-1}\right|_{1, h}^{2} \leq & \tau\left|e_{\text {mid }}^{n-1}\right|_{1, h}^{2}+\tau\left|e_{\text {mid }}^{n-2}\right|_{1, h}^{2}+C_{\delta_{\star}} \tau\left|\eta^{n}-\eta^{n-1}\right|_{1, h}\left(\left|e_{\text {mid }}^{n}\right|_{1, h}+\left|e_{\text {mid }}^{n-2}\right|_{1, h}\right) \\
& +C_{\delta_{\star}} \tau^{2}\left(\tau^{2}+h^{2}\right)\left(\left|e_{\text {mid }}^{n}\right|_{1, h}+\left|e_{\text {mid }}^{n-2}\right|_{1, h}\right) \\
\leq & \tau\left|e_{\text {mid }}^{n-1}\right|_{1, h}^{2}+\tau\left|e_{\text {mid }}^{n-2}\right|_{1, h}^{2}+\left|\eta^{n}-\eta^{n-1}\right|_{1, h}^{2}+C_{\delta_{\star}} \tau^{2}\left(\left|e_{\text {mid }}^{n}\right|_{1, h}+\left|e_{\text {mid }}^{n-2}\right|_{1, h}\right)^{2} \\
& +C_{\delta_{\star}} \tau^{2}\left[\left(\left|e_{\text {mid }}^{n}\right|_{1, h}+\left|e_{\text {mid }}^{n-2}\right|_{1, h}\right)^{2}+\left(\tau^{2}+h^{2}\right)^{2}\right] \\
\leq & \tau\left|e_{\text {mid }}^{n-1}\right|_{1, h}^{2}+\tau\left|e_{\text {mid }}^{n-2}\right|_{1, h}^{2}+\left|\eta^{n}-\eta^{n-1}\right|_{1, h}^{2} \\
& +C_{\delta_{\star}}\left[\tau^{2}\left(\left|e_{\text {mid }}^{n}\right|_{1, h}^{2}+\left|e_{\text {mid }}^{n-2}\right|_{1, h}^{2}\right)+\tau^{2}\left(\tau^{2}+h^{2}\right)^{2}\right]
\end{aligned}
$$

for $n=2, \ldots, N-1$. Combining (4.48) and (4.51), we conclude that there exists a positive constant $\mathrm{C}_{\delta_{\star}}^{\text {BR,VII }}$ such that:

$$
\left(1-\mathrm{C}_{\delta_{\star}, \mathrm{VII}}^{\mathrm{BR}} \tau\right) \mathrm{E}_{\star}^{n+1} \leq\left(1+\mathrm{C}_{\delta_{\star}}^{\mathrm{BR}, \mathrm{VII}} \tau\right) \mathrm{E}_{\star}^{n}+C_{\delta_{\star}} \tau^{2}\left(\tau^{2}+h^{2}\right)^{2}, \quad n=2, \ldots, N-1,
$$

where

$$
\mathrm{E}_{\star}^{m}:=\left|\eta^{m}-\eta^{m-1}\right|_{1, h}^{2}+\tau\left|\eta^{m}\right|_{2, h}^{2}+\tau\left|e_{\text {mid }}^{m-1}\right|_{1, h}^{2}+\tau\left|e_{\text {mid }}^{m-2}\right|_{1, h}^{2}, \quad m=2, \ldots, N .
$$

Let us assume that $\tau \mathrm{C}_{\delta_{\star}}^{\mathrm{BR}, \mathrm{VIII}} \leq \frac{1}{2}$, where $\mathrm{C}_{\delta_{\star}}^{\mathrm{BR}, \mathrm{VIII}}:=\max \left\{\mathrm{C}_{\delta_{\star}}^{\mathrm{BR}, \mathrm{VII}}, \mathrm{C}_{\delta_{\star}}^{\mathrm{BR}, \mathrm{VI}}\right\}$. Then, employing a standard discrete Gronwall argument based on (4.52), it follows that

$$
\max _{2 \leq m \leq N} \mathrm{E}_{\star}^{m} \leq C_{\delta_{\star}}\left[\mathrm{E}_{\star}^{2}+\tau\left(\tau^{2}+h^{2}\right)^{2}\right]
$$


which, along with (4.53), yields

$$
\begin{aligned}
\tau\left[\max _{1 \leq m \leq N}\left|\eta^{m}\right|_{2, h}^{2}+\max _{0 \leq m \leq N-1}\left|e_{\text {mid }}^{m}\right|_{1, h}^{2}\right] \leq & C_{\delta_{\star}}\left[\left|\eta^{2}-\eta^{1}\right|_{1, h}^{2}+\tau\left|\eta^{2}\right|_{2, h}^{2}+\tau\left|\eta^{1}\right|_{2, h}^{2}\right. \\
& \left.+\tau\left|e_{\text {mid }}^{1}\right|_{1, h}^{2}+\tau\left|e_{\text {mid }}^{0}\right|_{1, h}^{2}+\tau\left(\tau^{2}+h^{2}\right)^{2}\right] .
\end{aligned}
$$

Combining (4.48) (with $n=0)$ and (3.19), we obtain

$$
\begin{aligned}
\tau\left|\eta^{1}\right|_{2, h}^{2} & \leq C_{\delta_{\star}}\left[\tau\left|\eta^{0}\right|_{2, h}^{2}+\tau^{2}\left|e_{\text {mid }}^{0}\right|_{1, h}^{2}+\tau^{2}\left(\tau^{2}+h^{2}\right)^{2}\right] \\
& \leq C_{\delta_{\star}}\left[\tau h^{4}+\tau^{2}\left|e_{\text {mid }}^{0}\right|_{1, h}^{2}+\tau^{2}\left(\tau^{2}+h^{2}\right)^{2}\right] \\
& \leq C_{\delta_{\star}}\left[\tau^{2}\left|e_{\text {mid }}^{0}\right|_{1, h}^{2}+\tau\left(\tau^{2}+h^{2}\right)^{2}\right]
\end{aligned}
$$

Using (4.48) (with $n=1$ ) and (4.55) we have

$$
\begin{aligned}
\left|\eta^{2}-\eta^{1}\right|_{1, h}^{2}+\tau\left|\eta^{2}\right|_{2, h}^{2} & \leq C_{\delta_{\star}}\left[\tau\left|\eta^{1}\right|_{2, h}^{2}+\tau^{2}\left|e_{\text {mid }}^{1}\right|_{1, h}^{2}+\tau^{2}\left(\tau^{2}+h^{2}\right)^{2}\right] \\
& \leq C_{\delta_{\star}}\left[\tau^{2}\left|e_{\text {mid }}^{1}\right|_{1, h}^{2}+\tau^{2}\left|e_{\text {mid }}^{0}\right|_{1, h}^{2}+\tau\left(\tau^{2}+h^{2}\right)^{2}\right]
\end{aligned}
$$

Also, from (4.28) (with $n=1$ ), (2.7) (with $\mathfrak{g}=g \circ \mathfrak{n}_{\delta_{\star}}$ ), (2.3), (3.19), (2.6) and (3.10), we conclude that

$$
\begin{aligned}
\left|e_{\text {mid }}^{1}\right|_{1, h}^{2} & \leq\left[\left|e_{\text {mid }}^{0}\right|_{1, h}+2\left|g\left(\mathfrak{n}_{\delta_{\star}}\left(u^{1}\right)\right)-g\left(\mathfrak{n}_{\delta_{\star}}\left(V_{\delta_{\star}}^{1}\right)\right)\right|_{1, h}+2\left|\mathrm{r}^{1}\right|_{1, h}\right]^{2} \\
& \leq C_{\delta_{\star}}\left(\left|e_{\text {mid }}^{0}\right|_{1, h}^{2}+\left|e^{1}\right|_{1, h}^{2}+\tau^{4}\right) \\
& \leq C_{\delta_{\star}}\left(\left|e_{\text {mid }}^{0}\right|_{1, h}^{2}+\left|\rho^{1}\right|_{1, h}^{2}+\left|\eta^{1}\right|_{1, h}^{2}+\tau^{4}\right) \\
& \leq C_{\delta_{\star}}\left[\left|e_{\text {mid }}^{0}\right|_{1, h}^{2}+\left|\eta^{1}\right|_{2, h}^{2}+\left(h^{2}+\tau^{2}\right)^{2}\right] \\
& \leq C_{\delta_{\star}}\left[\left|e_{\text {mid }}^{0}\right|_{1, h}^{2}+\left(h^{2}+\tau^{2}\right)^{2}\right]
\end{aligned}
$$

Since $e^{0}=0$, using (3.19), we get

$$
\begin{aligned}
\max _{0 \leq m \leq N}\left|e^{m}\right|_{2, h}^{2} & \leq \max _{1 \leq m \leq N}\left(\left|\rho^{m}\right|_{2, h}+\left|\eta^{m}\right|_{2, h}\right)^{2} \\
& \leq C h^{4}+\max _{1 \leq m \leq N}\left|\eta^{m}\right|_{2, h}^{2} .
\end{aligned}
$$

Thus, from (4.54) to (4.58), it follows that

$$
\max _{0 \leq m \leq N}\left|e^{m}\right|_{2, h}^{2}+\max _{0 \leq m \leq N-1}\left|e_{\text {mid }}^{m}\right|_{1, h}^{2} \leq C_{\delta_{\star}}\left[\left|e_{\text {mid }}^{0}\right|_{1, h}^{2}+\left(\tau^{2}+h^{2}\right)^{2}\right] .
$$

Finally, (4.13) follows, easily, from (4.59) and (4.21).

Next, we investigate the influence on the convergence rate of a different construction of $\Phi_{\delta_{\star}}^{\frac{1}{2}}($ see $[4,5])$.

Corollary 4.5. Let $g \in C^{4}(\mathbb{R}), u \in C_{t, x}^{3,0}(Q), \partial_{t}^{\ell} u \in C_{t, x}^{0,1}(Q)$ for $\ell=1,2,3$, and $\partial_{t} u \in C_{t, x}^{0,4}(Q)$. Also, let $u_{\max }:=\max _{Q}|u|, g_{\max }:=\max _{Q}|g(u)|, \delta_{\star} \geq \max \left\{u_{\max }, g_{\max }\right\}, C_{\delta_{\star}}^{\mathrm{BR}, \mathrm{I}}:=\frac{1}{4} \max _{\mathbb{R}}\left|\mathfrak{n}_{\delta}\right|$ and $\tau \mathrm{C}_{\delta_{\star}}^{\mathrm{BR}, \mathrm{I}} \leq \frac{1}{2}$. If

$$
\Phi_{\delta_{\star}}^{\frac{1}{2}}:=g\left(u^{0}\right)
$$

then, there exist constants $\mathrm{C}_{\delta_{\star}}^{\mathrm{B}, 1} \geq \mathrm{C}_{\delta_{\star}}^{\mathrm{BR}, 1}$ and $\mathrm{C}_{\delta_{\star}}^{\mathrm{B}, 2}>0$, independent of $\tau$ and $h$, such that: if $\tau \mathrm{C}_{\delta_{\star}}^{\mathrm{B}, 1} \leq \frac{1}{2}$, then

$$
\max _{0 \leq m \leq N-1}\left|g\left(u^{m+\frac{1}{2}}\right)-\Phi_{\delta_{\star}}^{m+\frac{1}{2}}\right|_{1, h}+\max _{0 \leq m \leq N}\left|u^{m}-V_{\delta_{\star}}^{m}\right|_{2, h} \leq \mathrm{C}_{\delta_{\star}}^{\mathrm{B}, 2}\left(\tau+h^{2}\right) .
$$


Proof. Since $\delta_{\star} \geq u_{\max }$, estimate (4.61) is a simple consequence of (4.60), (2.7) (with $\mathfrak{g}=g \circ \mathfrak{n}_{\delta_{\star}}$ ) and (4.59).

\subsection{Convergence of the (BRFD) method}

Theorem 4.6. Let $g \in C^{4}(\mathbb{R}), u \in C_{t, x}^{3,0}(Q), \partial_{t}^{\ell} u \in C_{t, x}^{0,1}(Q)$ for $\ell=1,2,3$, and $\partial_{t} u \in C_{t, x}^{0,4}(Q)$. Also, let $u_{\max }:=\max _{Q}|u|, g_{\max }:=\max _{Q}|g \circ u|, g_{\max }^{0}:=\max _{\mathcal{I}}\left|g \circ u_{0}\right|, \delta_{\star} \geq 2 \max \left\{u_{\max }, g_{\max }\right\}, C_{\delta_{\star}}^{\mathrm{BR}, 1}:=\frac{1}{4} \max _{\mathbb{R}}\left|\mathfrak{n}_{\delta}\right|, \mathrm{C}_{\delta_{\star}}^{\mathrm{BCV}, 1}$, $\mathrm{C}_{\delta_{\star}}^{\mathrm{BCV}, 2}, \mathrm{C}_{\delta_{\star}}^{\mathrm{BCV}, 3}$ and $\mathrm{C}_{\delta_{\star}}^{\mathrm{BCV}, 4}$ be the constants specified in Theorem 4.4, where $\mathrm{C}_{\delta_{\star}}^{\mathrm{BCV}, 1} \geq \mathrm{C}_{\delta_{\star}}^{\mathrm{BR}, 1}$. If $\tau \mathrm{C}_{\delta_{\star}}^{\mathrm{BCV}, 1} \leq \frac{1}{2}$ and

$$
\mathrm{C}_{\delta_{\star}}^{\mathrm{BCV}, 2} \sqrt{\mathrm{L}}\left(\tau^{2}+\tau^{\frac{1}{2}} h^{2}\right) \leq \frac{\delta_{\star}}{2}, \quad \max \left\{\mathrm{C}_{\delta_{\star}}^{\mathrm{BCV}, 3}, \mathrm{C}_{\delta_{\star}}^{\mathrm{BCV}, 4}\right\} \sqrt{\mathrm{L}}\left(\tau^{2}+h^{2}\right) \leq \frac{\delta_{\star}}{2},
$$

then, the method (BRFD) is well-defined and the following error estimates hold

$$
\left|u^{\frac{1}{2}}-U^{\frac{1}{2}}\right|_{1, h} \leq \mathrm{C}_{\delta_{\star}}^{\mathrm{BCV}, 2}\left(\tau^{2}+\tau^{\frac{1}{2}} h^{2}\right)
$$

and

$$
\max _{0 \leq m \leq N-1}\left|g\left(u^{m+\frac{1}{2}}\right)-\Phi^{m+\frac{1}{2}}\right|_{1, h}+\max _{0 \leq m \leq N}\left|u^{m}-U^{m}\right|_{2, h} \leq \max \left\{C_{\delta_{\star}}^{\mathrm{BCV}, 3}, \mathrm{C}_{\delta_{\star}}^{\mathrm{BCV}, 4}\right\}\left(\tau^{2}+h^{2}\right) .
$$

Proof. Since $\delta_{\star} \geq 2 \max \left\{g_{\max }, u_{\max }\right\} \geq g_{\max }^{0}$ and $\tau \mathrm{C}_{\delta_{\star}}^{\mathrm{BR}, 1} \leq \tau \mathrm{C}_{\delta_{\star}}^{\mathrm{BCV}, 1} \leq \frac{1}{2}$, Proposition 4.2 yields the existence and uniqueness of the (MBRFD) approximations when $\delta=\delta_{\star}$. Using the convergence estimates (4.11)-(4.13), the discrete Sobolev inequality (2.2) and the mesh size conditions (4.62) we have

$$
\begin{aligned}
\left|\Phi_{\delta_{\star}}^{n+\frac{1}{2}}\right|_{\infty, h} & \leq\left|g\left(u^{n+\frac{1}{2}}\right)-\Phi_{\delta_{\star}}^{n+\frac{1}{2}}\right|_{\infty, h}+\left|g\left(u^{n+\frac{1}{2}}\right)\right|_{\infty, h} \\
& \leq \sqrt{\mathrm{L}}\left|g\left(u^{n+\frac{1}{2}}\right)-\Phi_{\delta_{\star}}^{n+\frac{1}{2}}\right|_{1, h}+g_{\max } \\
& \leq \mathrm{C}_{\delta_{\star}}^{\mathrm{BCV}, 4} \sqrt{\mathrm{L}}\left(\tau^{2}+h^{2}\right)+\frac{\delta_{\star}}{2} \leq \delta_{\star}, \quad n=0, \ldots, N-1, \\
\left|V_{\delta_{\star}}^{n}\right|_{\infty, h} & \leq\left|u^{n}-V_{\delta_{\star}}^{n}\right|_{\infty, h}+\left|u^{n}\right|_{\infty, h} \\
& \leq \sqrt{\mathrm{L}}\left|u^{n}-V_{\delta_{\star}}^{n}\right|_{1, h}+u_{\max } \\
& \leq \mathrm{C}_{\delta_{\star}}^{\mathrm{BCV}, 3} \sqrt{\mathrm{L}}\left(\tau^{2}+h^{2}\right)+\frac{\delta_{\star}}{2} \leq \delta_{\star}, \quad n=1, \ldots, N-1,
\end{aligned}
$$

and

$$
\begin{aligned}
\left|V_{\delta_{\star}}^{\frac{1}{2}}\right|_{\infty, h} & \leq\left|u^{\frac{1}{2}}-V_{\delta_{\star}^{\frac{1}{2}}}\right|_{\infty, h}+\left|u^{\frac{1}{2}}\right|_{\infty, h} \\
& \leq \sqrt{\mathrm{L}}\left|u^{\frac{1}{2}}-V_{\delta_{\star}}^{\frac{1}{2}}\right|_{1, h}+u_{\max } \\
& \leq \mathrm{C}_{\delta_{\star}}^{\mathrm{BCv}, 2} \sqrt{\mathrm{L}}\left(\tau^{2}+\tau^{\frac{1}{2}} h^{2}\right)+\frac{\delta_{\star}}{2} \leq \delta_{\star},
\end{aligned}
$$

which, along with (4.1), yield

$$
\begin{aligned}
& \mathfrak{n}_{\delta_{\star}}\left(V_{\delta_{\star}}^{\ell}\right)=V_{\delta_{\star}}^{\ell}, \quad \ell=\frac{1}{2}, 1, \ldots, N-1, \\
& \mathfrak{n}_{\delta_{\star}}\left(\Phi_{\delta_{\star}}^{n+\frac{1}{2}}\right)=\Phi_{\delta_{\star}}^{n+\frac{1}{2}}, \quad n=0, \ldots, N-1 .
\end{aligned}
$$

Thus, for $\delta=\delta_{\star}$ the (MBRFD) approximations are (BRFD) approximations, i.e., (1.5)-(1.10) hold after replacing $U^{\frac{1}{2}}$ by $V_{\delta_{\star}}^{\frac{1}{2}}, U^{n}$ by $V_{\delta_{\star}}^{n}$ for $n=0, \ldots, N$, and $\Phi^{n+\frac{1}{2}}$ by $\Phi_{\delta_{\star}}^{n+\frac{1}{2}}$ for $n=0, \ldots, N-1$.

Let $U^{\frac{1}{2}},\left(U^{n}\right)_{n=0}^{N}$ and $\left(\Phi^{n+\frac{1}{2}}\right)_{n=0}^{N-1}$ be approximations derived by the (BRFD) method. Then, we introduce the errors $\mathfrak{q}^{\frac{1}{2}}:=V_{\delta_{\star}}^{\frac{1}{2}}-U^{\frac{1}{2}}, \mathfrak{q}^{n}:=V_{\delta_{\star}}^{n}-U^{n}$ for $n=0, \ldots, N$, and $\mathfrak{q}_{\text {mid }}^{n}:=\Phi_{\delta_{\star}}^{n+\frac{1}{2}}-\Phi^{n+\frac{1}{2}}$ for $n=0, \ldots, N-1$. 
Since $\tau \mathrm{C}_{\delta_{\star}}^{\mathrm{BR}, \mathrm{I}} \leq \frac{1}{2}$ and $\delta_{\star} \geq g_{\max } \geq g_{\max }^{0}$, Remark 4.3 and (1.5) yield $\mathfrak{q}^{0}=0, \mathfrak{q}^{\frac{1}{2}}=0$ and $\mathfrak{q}_{\text {mid }}^{0}=0$. Now, we assume that for a given $m \in\{0, \ldots, N-1\}$ it holds that $\mathfrak{q}^{m}=0$ and $\mathfrak{q}_{\text {mid }}^{m}=0$. Subtracting (1.10) from (4.7) (or (1.8) from (4.5) when $m=0$ ), and then using (4.65), we obtain

$$
\mathfrak{q}^{m+1}=\frac{\tau}{2} \Delta_{h} \mathfrak{q}^{m+1}+\frac{\tau}{2}\left[\mathfrak{n}_{\delta_{\star}}\left(\Phi_{\delta_{\star}}^{m+\frac{1}{2}}\right) \otimes \mathfrak{q}^{m+1}\right] .
$$

Next, taking the inner product $(\cdot, \cdot)_{0, h}$ with $\mathfrak{q}^{m+1}$ and then using (2.5), the Cauchy-Schwarz inequality, (4.1) and the definition of $C_{\delta_{\star}}^{\mathrm{BR}, \mathrm{I}}$, we get

$$
\begin{aligned}
0 & =\left\|\mathfrak{q}^{m+1}\right\|_{0, h}^{2}+\frac{\tau}{2}\left|\mathfrak{q}^{m+1}\right|_{1, h}^{2}-\frac{\tau}{2}\left(\mathfrak{n}_{\delta_{\star}}\left(\Phi_{\delta_{\star}}^{m+\frac{1}{2}}\right) \otimes \mathfrak{q}^{m+1}, \mathfrak{q}^{m+1}\right)_{0, h} \\
& \geq \frac{\tau}{2}\left|\mathfrak{q}^{m+1}\right|_{1, h}^{2}+2\left\|\mathfrak{q}^{m+1}\right\|_{0, h}^{2}\left(\frac{1}{2}-\frac{\tau}{4} \sup _{\mathbb{R}}\left|\mathfrak{n}_{\delta_{\star}}\right|\right) \\
& \geq \frac{\tau}{2}\left|\mathfrak{q}^{m+1}\right|_{1, h}^{2}+2\left\|\mathfrak{q}^{m+1}\right\|_{0, h}^{2}\left(\frac{1}{2}-\tau C_{\delta_{\star}}^{\mathrm{BR}, 1}\right) \\
& \geq \frac{\tau}{2}\left|\mathfrak{q}^{m+1}\right|_{1, h}^{2},
\end{aligned}
$$

which, obviously, yields that $\mathfrak{q}^{m+1}=0$. When $m \leq N-2$, observing that

$$
\mathfrak{q}_{\text {mid }}^{m+1}=2\left[g\left(V_{\delta_{\star}}^{m+1}\right)-g\left(U^{m+1}\right)\right]-\mathfrak{q}_{\text {mid }}^{m},
$$

we arrive at $\mathfrak{q}_{\text {mid }}^{m+1}=0$. The induction argument above, shows that, under our assumptions the (BRFD) approximations are only those derived from the (MBRFD) scheme when $\delta=\delta_{\star}$, and thus the error estimates (4.63) and (4.64) follow as a natural outcome of (4.11) and (4.13).

Theorem 4.7. Let $g \in C^{4}(\mathbb{R}), u \in C_{t, x}^{3,0}(Q), \partial_{t}^{\ell} u \in C_{t, x}^{0,1}(Q)$ for $\ell=1,2,3$, and $\partial_{t} u \in C_{t, x}^{0,4}(Q)$.

Also, let $u_{\max }:=\max _{Q}|u|, g_{\max }:=\max _{Q}|g(u)|, g_{\max }^{0}:=\max _{\mathcal{I}}\left|g \circ u_{0}\right|, \delta_{\star} \geq 2 \max \left\{u_{\max }, g_{\max }\right\}, C_{\delta_{\star}}^{\mathrm{BR}, 1}:=$ $\frac{1}{4} \max _{\mathbb{R}}\left|\mathfrak{n}_{\delta}\right|$, and $\mathrm{C}_{\delta_{\star}}^{\mathrm{B}, 1}$ and $\mathrm{C}_{\delta_{\star}}^{\mathrm{B}, 2}$ be the constants specified in Corollary 4.5, where $\mathrm{C}_{\delta_{\star}}^{\mathrm{B}, 1} \geq \mathrm{C}_{\delta_{\star}}^{\mathrm{BR}, \mathrm{I}}$. If $\tau \mathrm{C}_{\delta_{\star}}^{\mathrm{B}, 1} \leq \frac{1}{2}$ and

$$
\mathrm{C}_{\delta_{\star}}^{\mathrm{B}, 2} \max \left\{\mathrm{L}^{3 / 2}, \mathrm{~L}^{1 / 2}\right\}\left(\tau+h^{2}\right) \leq \frac{\delta_{\star}}{2},
$$

then, the method (BRFD) with $\Phi^{\frac{1}{2}}:=g\left(u^{0}\right)$ is well-defined and the following error estimates hold

$$
\max _{0 \leq m \leq N-1}\left|g\left(u^{m+\frac{1}{2}}\right)-\Phi^{m+\frac{1}{2}}\right|_{1, h} \leq C_{\delta_{\star}}^{\mathrm{B}, 2}\left(\tau+h^{2}\right)
$$

and, there exist constants $\mathrm{C}_{\delta_{\star}}^{\mathrm{B}, 3} \geq \mathrm{C}_{\delta_{\star}}^{\mathrm{B}, 1}$ and $\mathrm{C}_{\delta_{\star}, 4}^{\mathrm{B}, 4}$, such that: if $\tau \mathrm{C}_{\delta_{\star}}^{\mathrm{B}, 3} \leq \frac{1}{2}$, then

$$
\max _{0 \leq m \leq N}\left(\left\|u^{m}-U^{m}\right\|_{0, h}+\left|u^{m}-U^{m}\right|_{1, h}\right) \leq C_{\delta_{\star}}^{\mathrm{B}, 4}\left(\tau^{2}+h^{2}\right) .
$$

Proof. Since $\delta_{\star} \geq 2 \max \left\{g_{\max }, u_{\max }\right\} \geq g_{\max }^{0}$ and $\tau \mathrm{C}_{\delta_{\star}}^{\mathrm{BR}, 1} \leq \tau \mathrm{C}_{\delta_{\star}}^{\mathrm{B}, 1} \leq \frac{1}{2}$, Proposition 4.2 yields the existence and uniqueness of the (MBRFD) approximations when $\delta=\delta_{\star}$ and $\Phi_{\delta_{\star}}^{\frac{1}{2}}=g\left(u^{0}\right)$. Then, moving along the lines of the proof of Theorem 4.6, and using (4.67), (4.61), (2.2) and (2.6) we conclude that

$$
\max _{0 \leq m \leq N-1}\left|\Phi_{\delta_{\star}}^{m+\frac{1}{2}}\right|_{\infty, h} \leq \delta_{\star}, \quad \max _{1 \leq m \leq N-1}\left|V_{\delta_{\star}}^{m}\right|_{\infty, h} \leq \delta_{\star}
$$

and, in view of (4.1), we obtain

$$
\mathfrak{n}_{\delta_{\star}}\left(V_{\delta_{\star}}^{n}\right)=V_{\delta_{\star}}^{n}, \quad n=1, \ldots, N-1 \quad \text { and } \quad \mathfrak{n}_{\delta_{\star}}\left(\Phi_{\delta_{\star}}^{n+\frac{1}{2}}\right)=\Phi_{\delta_{\star}}^{n+\frac{1}{2}}, \quad n=0, \ldots, N-1 .
$$


In addition, we conclude that the (BRFD) approximations with $\Phi^{\frac{1}{2}}=g\left(u^{0}\right)$ are well-defined and are those derived from the (MBRFD) scheme when $\delta=\delta_{\star}$ and $\Phi_{\delta_{\star}}^{\frac{1}{2}}=g\left(u^{0}\right)$, and thus the error estimate (4.68) follow as a natural outcome of (4.61).

Recalling (4.22) and using (4.71), we have

$$
e^{n+1}-e^{n}=\frac{\tau}{2} \Delta_{h}\left(e^{n+1}+e^{n}\right)+\sum_{\kappa=1}^{3} \mathrm{Q}^{\kappa, n}, \quad n=0, \ldots, N-1,
$$

where $\mathrm{Q}^{1, n}:=\tau \mathrm{s}^{n+\frac{1}{2}}, \mathrm{Q}^{2, n}:=\frac{\tau}{2} \Phi_{\delta_{\star}}^{n+\frac{1}{2}} \otimes\left(e^{n+1}+e^{n}\right)$ and $\mathrm{Q}^{3, n}:=\frac{\tau}{2} e_{\text {mid }}^{n} \otimes\left(u^{n+1}+u^{n}\right)$.

Since $e^{0}=0$, after taking the $(\cdot, \cdot)_{0, h}$-inner product of $(4.72)$ with $\left(e^{n+1}+e^{n}\right)$, using $(2.5)$ and then summing with respect to $n$, we get

$$
\left\|e^{m}\right\|_{0, h}^{2} \leq \sum_{\kappa=1}^{3}\left[\sum_{n=0}^{m-1}\left(\mathbf{Q}^{\kappa, n}, e^{n+1}+e^{n}\right)_{0, h}\right], \quad m=1, \ldots, N .
$$

Let $m \in\{1 \ldots, N\}$. Using the Cauchy-Schwarz inequality, the arithmetic mean inequality, (3.8), (3.16) and (4.70), we have

$$
\begin{aligned}
\sum_{n=0}^{m-1}\left(\mathrm{Q}^{1, n}, e^{n+1}+e^{n}\right)_{0, h} & \leq \tau \sum_{n=0}^{m-1}\left(\left\|\mathrm{~s}^{n+\frac{1}{2}}-\mathrm{r}^{n+\frac{1}{2}}\right\|_{0, h}+\left\|\mathrm{r}^{n+\frac{1}{2}}\right\|_{0, h}\right)\left\|e^{n+1}+e^{n}\right\|_{0, h} \\
& \leq C \tau \sum_{n=0}^{m-1}\left(\tau^{2}+h^{2}\right)\left(\left\|e^{n+1}\right\|_{0, h}+\left\|e^{n}\right\|_{0, h}\right) \\
& \leq C\left(\tau^{2}+h^{2}\right)^{2}+2 \tau \sum_{n=0}^{m-1}\left(\left\|e^{n+1}\right\|_{0, h}^{2}+\left\|e^{n}\right\|_{0, h}^{2}\right) \\
& \leq C\left(\tau^{2}+h^{2}\right)^{2}+2 \tau\left\|e^{m}\right\|_{0, h}^{2}+4 \tau \sum_{n=0}^{m-1}\left\|e^{n}\right\|_{0, h}^{2}
\end{aligned}
$$

and

$$
\begin{aligned}
\sum_{n=0}^{m-1}\left(\mathrm{Q}^{2, n}, e^{n+1}+e^{n}\right)_{0, h} & \leq \frac{\tau}{2} \delta_{\star} \sum_{n=0}^{m-1}\left(\left\|e^{n+1}\right\|_{0, h}+\left\|e^{n}\right\|_{0, h}\right)^{2} \\
& \leq \tau \delta_{\star}\left\|e^{m}\right\|_{0, h}^{2}+\tau \delta_{\star} \sum_{n=0}^{m-1}\left\|e^{n}\right\|_{0, h}^{2} .
\end{aligned}
$$

Observing that

$$
\begin{aligned}
\sum_{n=0}^{m-1}\left(\mathrm{Q}^{3, n}, e^{n+1}+e^{n}\right)_{0, h}= & \frac{\tau}{2} \sum_{n=1}^{m}\left(e_{\text {mid }}^{n-1} \otimes\left(u^{n}+u^{n-1}\right), e^{n}\right)_{0, h}+\frac{\tau}{2} \sum_{n=0}^{m-1}\left(e_{\text {mid }}^{n} \otimes\left(u^{n+1}+u^{n}\right), e^{n}\right)_{0, h} \\
= & \frac{\tau}{2}\left(e_{\text {mid }}^{m-1} \otimes\left(u^{m}+u^{m-1}\right), e^{m}\right)_{0, h} \\
& +\frac{\tau}{2} \sum_{n=1}^{m-1}\left(\left(e_{\text {mid }}^{n-1}+e_{\text {mid }}^{n}\right) \otimes\left(u^{n}+u^{n-1}\right), e^{n}\right)_{0, h} \\
& +\frac{\tau}{2} \sum_{n=1}^{m-1}\left(e_{\text {mid }}^{n} \otimes\left(u^{n+1}-u^{n-1}\right), e^{n}\right)_{0, h}
\end{aligned}
$$


and then using the Cauchy-Schwarz inequality, the error bound (4.61) along with (2.3), (4.28), (3.10), (4.70) and the arithmetic mean inequality, we arrive at

$$
\begin{aligned}
\sum_{n=0}^{m-1}\left(\mathrm{Q}^{3, n}, e^{n+1}+e^{n}\right)_{0, h} \leq & C_{\delta_{\star}}\left(\tau^{2}+\tau h^{2}\right)\left\|e^{m}\right\|_{0, h}+C_{\delta_{\star}} \tau \sum_{n=1}^{m-1}\left(\tau^{2}+\tau h^{2}\right)\left\|e^{n}\right\|_{0, h} \\
& +C \tau \sum_{n=1}^{m-1}\left(\left\|\mathrm{r}^{n}\right\|_{0, h}+\left\|e^{n}\right\|_{0, h}\right)\left\|e^{n}\right\|_{0, h} \\
\leq & C_{\delta_{\star}}\left(\tau^{2}+\tau h^{2}\right)^{2}+\frac{1}{2}\left\|e^{m}\right\|_{0, h}^{2}+C_{\delta_{\star}} \tau \sum_{m=0}^{m-1}\left\|e^{n}\right\|_{0, h}^{2} .
\end{aligned}
$$

From (4.73) to (4.76), we conclude that there exists a constant $C_{\delta_{\star}}^{\mathrm{B}, \star} \geq \mathrm{C}_{\delta_{\star}}^{\mathrm{B}, 1}$ such that

$$
\left(1-\tau \mathrm{C}_{\delta_{\star} \mathrm{B}, \star}\right)\left\|e^{m}\right\|_{0, h}^{2} \leq C_{\delta_{\star}}\left(\tau^{2}+h^{2}\right)+C_{\delta_{\star}} \tau \sum_{n=0}^{m-1}\left\|e^{n}\right\|_{0, h}^{2}, \quad m=1, \ldots, N .
$$

Assuming that $\tau \mathrm{C}_{\delta_{\star}}^{\mathrm{B}, \star} \leq \frac{1}{2}$, and applying a discrete Gronwall argument (4.77) yields

$$
\max _{0 \leq m \leq N}\left\|e^{m}\right\|_{0, h} \leq C_{\delta_{\star}}\left(\tau^{2}+h^{2}\right) .
$$

Since $e^{0}=0$, after taking the $(\cdot, \cdot)_{0, h}$-inner product of (4.72) with $\Delta_{h}\left(e^{n+1}+e^{n}\right)$, using (2.5), and then summing with respect to $n$, we get

$$
\left|e^{m}\right|_{1, h}^{2}+\frac{\tau}{2} \sum_{n=0}^{m-1}\left|e^{n+1}+e^{n}\right|_{2, h} \leq-\sum_{\kappa=1}^{3}\left[\sum_{n=0}^{m-1}\left(\mathrm{Q}^{\kappa, n}, \Delta_{h}\left(e^{n+1}+e^{n}\right)\right)_{0, h}\right], \quad m=1, \ldots, N .
$$

Let $m \in\{1 \ldots, N\}$. Using the Cauchy-Schwarz inequality, (3.8), (3.16), (4.70), (4.78) and the arithmetic mean inequality, we have

$$
\begin{aligned}
-\sum_{n=0}^{m-1}\left(\mathrm{Q}^{1, n}, \Delta_{h}\left(e^{n+1}+e^{n}\right)\right)_{0, h} & \leq \tau \sum_{n=0}^{m-1}\left(\left\|\mathbf{s}^{n+\frac{1}{2}}-\mathrm{r}^{n+\frac{1}{2}}\right\|_{0, h}+\left\|\mathrm{r}^{n+\frac{1}{2}}\right\|_{0, h}\right)\left|e^{n+1}+e^{n}\right|_{2, h} \\
& \leq C \tau \sum_{n=0}^{m-1}\left(\tau^{2}+h^{2}\right)\left|e^{n+1}+e^{n}\right|_{2, h} \\
& \leq C\left(\tau^{2}+h^{2}\right)^{2}+\frac{\tau}{4} \sum_{n=0}^{m-1}\left|e^{n+1}+e^{n}\right|_{2, h}^{2}
\end{aligned}
$$

and

$$
\begin{aligned}
-\sum_{n=0}^{m-1}\left(\mathrm{Q}^{2, n}, \Delta_{h}\left(e^{n+1}+e^{n}\right)\right)_{0, h} & \leq \frac{\tau}{2} \delta_{\star} \sum_{n=0}^{m-1}\left|\Phi_{\delta_{\star}}^{n+\frac{1}{2}}\right|_{\infty, h}\left\|e^{n+1}+e^{n}\right\|_{0, h}\left|e^{n+1}+e^{n}\right|_{2, h} \\
& \leq C_{\delta_{\star}} \tau \sum_{n=0}^{m-1}\left(\tau^{2}+h^{2}\right)\left|e^{n+1}+e^{n}\right|_{2, h} \\
& \leq C\left(\tau^{2}+h^{2}\right)^{2}+\frac{\tau}{4} \sum_{n=0}^{m-1}\left|e^{n+1}+e^{n}\right|_{2, h}^{2} .
\end{aligned}
$$


Using (2.5) and rearranging the terms in the sums, we get

$$
\begin{aligned}
-\sum_{n=0}^{m-1}\left(\mathrm{Q}^{3, n}, \Delta_{h}\left(e^{n+1}+e^{n}\right)\right)_{0, h}= & -\frac{\tau}{2} \sum_{n=1}^{m}\left(e_{\text {mid }}^{n-1} \otimes\left(u^{n}+u^{n-1}\right), \Delta_{h} e^{n}\right)_{0, h} \\
& -\frac{\tau}{2} \sum_{n=0}^{m-1}\left(e_{\text {mid }}^{n} \otimes\left(u^{n+1}+u^{n}\right), \Delta_{h} e^{n}\right)_{0, h} \\
= & \mathcal{T}_{1}+\mathcal{T}_{2}+\mathcal{T}_{3},
\end{aligned}
$$

where

$$
\begin{aligned}
& \mathcal{T}_{1}:=\frac{\tau}{2}\left(\left(\delta_{h}\left(e_{\text {mid }}^{m-1} \otimes\left(u^{m}+u^{m-1}\right)\right), \delta_{h} e^{m}\right)\right)_{0, h} \\
& \mathcal{T}_{2}:=\frac{\tau}{2} \sum_{n=1}^{m-1}\left(\left(\delta_{h}\left(\left(e_{\text {mid }}^{n-1}+e_{\text {mid }}^{n}\right) \otimes\left(u^{n}+u^{n-1}\right)\right), \delta_{h} e^{n}\right)\right)_{0, h} \\
& \mathcal{T}_{3}:=\frac{\tau}{2} \sum_{n=1}^{m-1}\left(\left(\delta_{h}\left(e_{\text {mid }}^{n} \otimes\left(u^{n+1}-u^{n-1}\right)\right), \delta_{h} e^{n}\right)_{0, h} .\right.
\end{aligned}
$$

Now, we apply the Cauchy-Schwarz inequality, (2.3), (4.61), (4.28), (3.10), (2.7) (with $\mathfrak{g}=g \circ \mathfrak{n}_{\delta_{\star}}$ ) and the arithmetic mean inequality, to obtain

$$
\begin{aligned}
\mathcal{T}_{1} & \leq \frac{\tau}{2}\left[\left|e_{\text {mid }}^{m-1}\right|_{1, h}\left|u^{m+1}+u^{m}\right|_{\infty, h}+\left\|e_{\text {mid }}^{m-1}\right\|_{0, h}\left\|\delta_{h}\left(u^{m+1}+u^{m}\right)\right\| \|_{\infty, h}\right]\left|e^{m}\right|_{1, h} \\
& \leq C \tau\left|e_{\text {mid }}^{m-1}\right|_{1, h}\left|e^{m}\right|_{1, h} \\
& \leq C\left(\tau^{2}+\tau h^{2}\right)\left|e^{m}\right|_{1, h} \\
& \leq C\left(\tau^{2}+\tau h^{2}\right)^{2}+\frac{1}{3}\left|e^{m}\right|_{1, h}^{2} \\
\mathcal{T}_{2} & \leq \frac{\tau}{2} \sum_{n=1}^{m-1}\left|e_{\text {mid }}^{n-1}+e_{\text {mid }}^{n}\right|_{1, h}\left|e^{n}\right|_{1, h} \\
& \leq \tau \sum_{n=1}^{m-1}\left[\left|g\left(u^{n}\right)-g\left(V_{\delta_{\star}}^{n}\right)\right|_{1, h}+\left|\mathrm{r}^{n}\right|_{1, h}\right]\left|e^{n}\right|_{1, h} \\
& \leq C_{\delta_{\star}} \tau \sum_{n=1}^{m-1}\left(\left|e^{n}\right|_{1, h}+\tau^{2}\right)\left|e^{n}\right|_{1, h} \\
& \leq C_{\delta_{\star}} \tau^{4}+C_{\delta_{\star}} \tau \sum_{n=1}^{m-1}\left|e^{n}\right|_{1, h}^{2}
\end{aligned}
$$

and

$$
\begin{aligned}
\mathcal{T}_{3} & \leq \frac{\tau}{2} \sum_{n=1}^{m-1}\left[\left|e_{\text {mid }}^{n}\right|_{1, h}\left|u^{n+1}-u^{n-1}\right|_{\infty, h}+\left\|e_{\text {mid }}^{n} \mid\right\|_{0, h}\left\|\delta_{h}\left(u^{n+1}-u^{n-1}\right)\right\| \|_{\infty, h}\right]\left|e^{n}\right|_{1, h} \\
& \leq C \tau^{2} \sum_{n=1}^{m-1}\left|e_{\text {mid }}^{n}\right|_{1, h}\left|e^{n}\right|_{1, h} \\
& \leq C_{\delta_{\star}} \tau \sum_{n=1}^{m-1}\left(\tau^{2}+\tau h^{2}\right)\left|e^{n}\right|_{1, h} \\
& \leq C_{\delta_{\star}}\left(\tau^{2}+\tau h^{2}\right)^{2}+C_{\delta_{\star}} \tau \sum_{n=1}^{m-1}\left|e^{n}\right|_{1, h}^{2} .
\end{aligned}
$$


From (4.79) to (4.85), we conclude that there exists a constant $C_{\delta_{\star}}^{B, \star \star} \geq C_{\delta_{\star}}^{B, \star}$ such that

$$
\left(1-\tau \mathrm{C}_{\delta_{\star}}^{\mathrm{B}, \star \star}\right)\left|e^{m}\right|_{1, h}^{2} \leq C_{\delta_{\star}}\left(\tau^{2}+h^{2}\right)+C_{\delta_{\star}} \tau \sum_{n=0}^{m-1}\left\|e^{n}\right\|_{0, h}^{2}, \quad m=1, \ldots, N .
$$

Assuming that $\tau \mathrm{C}_{\delta_{\star}, \star \star}^{\mathrm{B}} \leq \frac{1}{2}$, and applying a discrete Gronwall argument (4.86) yields

$$
\max _{0 \leq m \leq N}\left|e^{m}\right|_{1, h} \leq C_{\delta_{\star}}\left(\tau^{2}+h^{2}\right) .
$$

Thus, (4.69) follows easily from (4.78) and (4.87).

\section{NumericAl RESUlts}

We implemented the finite difference method (BRFD) in a FORTRAN 77 program which uses double precision real arithmetic and employs the subroutine dgtsl from LINPACK (see [7]) to solve the resulting tridiagonal linear systems of algebraic equations. Also, the program gives to its user the option to set $\Phi^{\frac{1}{2}}=g\left(u^{0}\right)$, or $\Phi^{\frac{1}{2}}=g\left(U^{\frac{1}{2}}\right)$ after computing $U^{\frac{1}{2}}$ via (1.6). When the exact solution to the problem is available, the program computes the following discrete $L_{t}^{\infty}\left(H_{x}^{1}\right)$-norm approximation errors:

$$
E_{\Phi}(N, J+1):=\max _{0 \leq n \leq N-1}\left|\Phi^{n+\frac{1}{2}}-g\left(u^{n+\frac{1}{2}}\right)\right|_{1, h}
$$

and

$$
E_{U}(N, J+1):=\max \left\{\left|U^{\frac{1}{2}}-u^{\frac{1}{2}}\right|_{1, h}, \max _{0 \leq n \leq N}\left|U^{n}-u^{n}\right|_{1, h}\right\}
$$

when $\Phi^{\frac{1}{2}}=g\left(U^{\frac{1}{2}}\right)$, or

$$
E_{U}^{\star}(N, J+1):=\max _{0 \leq n \leq N}\left|U^{n}-u^{n}\right|_{1, h}
$$

when $\Phi^{\frac{1}{2}}=g\left(u^{0}\right)$. Finally, letting $N$ be proportional to $(J+1)$ (i.e., $N=q(J+1)$ for a given $q \in \mathbb{Q}$ ), we compute the experimental order of convergence for successive values values $J_{1}$ and $J_{2}$ of $J$, using the formula

$$
\log \left(E\left(q\left(J_{1}+1\right),\left(J_{1}+1\right)\right) / E\left(q\left(J_{2}+1\right),\left(J_{2}+1\right)\right)\right) / \log \left(\left(J_{2}+1\right) /\left(J_{1}+1\right)\right),
$$

where $E=E_{U}, E_{\Phi}$ or $E_{U}^{\star}$.

Let us now consider the problem (1.1)-(1.4) with: $g(x)=1-x^{2}$ and load $f$ such that the function $u(t, x)=$ $e^{\frac{t}{10}}\left(x-x_{b}\right) \sin \left(\pi\left(x-x_{a}\right)\right)$ to be its exact solution. In the first set of numerical experiments we choose $T=1$, $\left[x_{a}, x_{b}\right]=[0,1],(N, J+1)=(\nu, \nu)$ for $\nu=20,40,80,160,320,640,1280, \Phi^{\frac{1}{2}}=g\left(U^{\frac{1}{2}}\right)$ and compute the approximation errors $E_{U}(\nu, \nu)$ and $E_{\Phi}(\nu, \nu)$. The results we obtain are shown on Table 1 and confirm that the experimental order of convergence with respect to $\frac{1}{\nu}$ is equal to 2 , which is in agreement with the results of the convergence analysis (see Thm. 4.6). In the second set of numerical experiments we choose $T=1,\left[x_{a}, x_{b}\right]=[0,1]$, $(N, J+1)=(\nu, \nu)$ for $\nu=20,40,80,160,320,640,1280, \Phi^{\frac{1}{2}}=g\left(u^{0}\right)$ and compute the approximation errors $E_{U}^{\star}(\nu, \nu)$ and $E_{\Phi}(\nu, \nu)$. We display the results obtained on Table 2, where it is obvious that the experimental order of convergence with respect to $\frac{1}{\nu}$ is equal to 1 for the error approximating $g(u)$ at the intermediate time nodes and is equal to 2 for the error approximating $u$ at the time nodes. Again, the behaviour of the method is in agreement with the results of the convergence analysis (see Thm. 4.7). 
TABLE 1. Discrete $H^{1}$ norm convergence when with $\Phi^{\frac{1}{2}}=g\left(U^{\frac{1}{2}}\right)$.

\begin{tabular}{llllll}
\hline \hline$\nu$ & $E_{U}(\nu, \nu)$ & Rate & $\nu$ & $E_{\Phi}(\nu, \nu)$ & Rate \\
\hline 20 & $4.087(-3)$ & - & 20 & $5.509(-3)$ & - \\
40 & $1.025(-3)$ & 1.994 & 40 & $1.255(-3)$ & 2.133 \\
80 & $2.565(-4)$ & 1.999 & 80 & $3.152(-4)$ & 1.999 \\
160 & $6.414(-5)$ & 1.999 & 160 & $7.921(-5)$ & 1.999 \\
320 & $1.603(-5)$ & 1.999 & 320 & $1.983(-5)$ & 1.999 \\
640 & $4.009(-6)$ & 2.000 & 640 & $4.961(-6)$ & 1.999 \\
1280 & $1.002(-6)$ & 1.999 & 1280 & $1.240(-6)$ & 1.999 \\
\hline
\end{tabular}

TABLE 2. Discrete $H^{1}$ norm convergence with $\Phi^{\frac{1}{2}}=g\left(u^{0}\right)$.

\begin{tabular}{llllll}
\hline \hline$\nu$ & $E_{U}^{\star}(\nu, \nu)$ & Rate & $\nu$ & $E_{\Phi}(\nu, \nu)$ & Rate \\
\hline 20 & $4.210(-3)$ & - & 20 & $1.673(-2)$ & - \\
40 & $1.049(-3)$ & 2.004 & 40 & $5.995(-3)$ & 1.480 \\
80 & $2.623(-4)$ & 2.000 & 80 & $2.559(-3)$ & 1.228 \\
160 & $6.559(-5)$ & 1.999 & 160 & $1.189(-3)$ & 1.105 \\
320 & $1.640(-5)$ & 1.999 & 320 & $5.748(-4)$ & 1.049 \\
640 & $4.101(-6)$ & 1.999 & 640 & $2.827(-4)$ & 1.023 \\
1280 & $1.025(-6)$ & 1.999 & 1280 & $1.402(-4)$ & 1.011 \\
\hline
\end{tabular}

\section{Conclusions}

Coupling the Besse Relaxation Scheme [4] with a finite difference scheme for space discretization, we arrive at a linearly implicit, fully discrete method for the approximation of the solution to a semilinear heat equation. For the proposed method, we derive optimal, second order error estimates in various discrete norms. Investigating the first order initial choice $\Phi^{\frac{1}{2}}=g\left(u^{0}\right)$, we develop a re-estimation argument that leads to a new, second order error estimate at the time nodes and to a first order error estimate at the intermediate nodes, which are in agreement with results obtained from numerical experiments.

\section{Appendix A.}

Proof of Lemma 2.2. Let $v, w \in \mathrm{X}_{h}^{\circ}$. First, we define $\mathfrak{a}^{s}, \mathfrak{b}^{s} \in \mathrm{G}_{h}$ by $\mathfrak{a}_{j}^{s}:=s v_{j+1}+(1-s) v_{j}$ and $\mathfrak{b}_{j}^{s}:=$ $s w_{j+1}+(1-s) w_{j}$ for $j=0, \ldots, J$ and $s \in[0,1]$. Then, we use the mean value theorem, to conclude that

$$
\delta_{h}(\mathfrak{g}(v)-\mathfrak{g}(w))=\mathcal{L}^{A}+\mathcal{L}^{B}
$$

where $\mathcal{L}^{A}, \mathcal{L}^{B} \in \mathrm{G}_{h}$ given by $\mathcal{L}_{j}^{A}:=\left(\delta_{h}(v-w)\right)_{j} \int_{0}^{1} \mathfrak{g}^{\prime}\left(\mathfrak{a}_{j}^{s}\right) \mathrm{d} s$ and $\mathcal{L}_{j}^{B}:=\delta_{h} w_{j} \int_{0}^{1}\left[\mathfrak{g}^{\prime}\left(\mathfrak{a}_{j}^{s}\right)-\mathfrak{g}^{\prime}\left(\mathfrak{b}_{j}^{s}\right)\right] \mathrm{d} s$ for $j=0, \ldots, J$. Observing that $\left|\mathcal{L}_{j}^{A}\right| \leq \sup _{\mathbb{R}}\left|\mathfrak{g}^{\prime}\right|\left|\left(\delta_{h}(v-w)\right)_{j}\right|$ for $j=0, \ldots, J$, and

$$
\begin{aligned}
\left|\mathcal{L}_{j}^{B}\right| & \leq\left|\left(\delta_{h} w\right)_{j}\right| \sup _{\mathbb{R}}\left|\mathfrak{g}^{\prime \prime}\right|\left|\int_{0}^{1}\left[s\left(v_{j+1}-w_{j+1}\right)+(1-s)\left(v_{j}-w_{j}\right)\right] \mathrm{d} s\right| \\
& \leq \frac{1}{2}\left|\left(\delta_{h} w\right)_{j}\right| \sup _{\mathbb{R}}\left|\mathfrak{g}^{\prime \prime}\right|\left(\left|v_{j+1}-w_{j+1}\right|+\left|v_{j}-w_{j}\right|\right), \quad j=0, \ldots, J
\end{aligned}
$$


we, easily, arrive at

$$
\begin{aligned}
\left\|\mathcal{L}^{A}\right\|_{0, h} & \leq \sup _{\mathbb{R}}\left|\mathfrak{g}^{\prime}\right|\left\|\delta_{h}(v-w)\right\|_{0, h}, \\
\left\|\mathcal{L}^{B}\right\|_{0, h} & \leq\left\|\delta_{h} w\right\|_{\infty, h} \sup _{\mathbb{R}}\left|\mathfrak{g}^{\prime \prime}\right|\|v-w\|_{0, h} .
\end{aligned}
$$

Thus, (2.7) follows as a simple consequence of (A.1)-(A.3).

\section{Appendix B.}

Proof of Lemma 2.3. Let $v^{a}, v^{b}, z^{a}, z^{b} \in \mathrm{X}_{h}^{\circ}$. We simplify the notation, first, by defining $\mathfrak{a}^{s}, \mathfrak{b}^{s} \in \mathrm{X}_{h}^{\circ}$ by $\mathfrak{a}^{s}:=$ $s v^{a}+(1-s) v^{b}$ and $\mathfrak{b}^{s}:=s z^{a}+(1-s) z^{b}$ for $s \in[0,1]$, and then, by introducing $\mathfrak{f} \in \mathrm{X}_{h}$ by $\mathfrak{f}:=\int_{0}^{1} \mathfrak{g}^{\prime}\left(\mathfrak{a}^{s}\right) \mathrm{d} s$ and $\mathfrak{t} \in \mathrm{X}_{h}^{\circ}$ by $\mathfrak{t}:=\int_{0}^{1}\left[\mathfrak{g}^{\prime}\left(\mathfrak{a}^{s}\right)-\mathfrak{g}^{\prime}\left(\mathfrak{b}^{s}\right)\right] \mathrm{d} s$. Also, we set $e^{a}:=v^{a}-z^{a}$ and $e^{b}:=v^{b}-z^{b}$.

Part I. First, we use the definition of $\mathfrak{f}$ and the mean value theorem, to get

$$
|\mathfrak{f}|_{\infty, h} \leq \sup _{\mathbb{R}}\left|\mathfrak{g}^{\prime}\right|
$$

and

$$
\begin{aligned}
\left|\delta_{h} \mathfrak{f}_{j}\right| & \leq \frac{1}{h} \int_{0}^{1}\left|\mathfrak{g}^{\prime}\left(\mathfrak{a}_{j+1}^{s}\right)-\mathfrak{g}^{\prime}\left(\mathfrak{a}_{j}^{s}\right)\right| \mathrm{d} s \\
& \leq \sup _{\mathbb{R}}\left|\mathfrak{g}^{\prime \prime}\right| \int_{0}^{1}\left|s \delta_{h} v_{j}^{a}+(1-s) \delta_{h} v_{j}^{b}\right| \mathrm{d} s \\
& \leq \frac{1}{2} \sup _{\mathbb{R}}\left|\mathfrak{g}^{\prime \prime}\right|\left(\left|\delta_{h} v_{j}^{a}\right|+\left|\delta_{h} v_{j}^{b}\right|\right), \quad j=0, \ldots, J
\end{aligned}
$$

which, obviously, yields

$$
|\mathfrak{f}|_{1, h} \leq \frac{1}{2} \sup _{\mathbb{R}}\left|\mathfrak{g}^{\prime \prime}\right|\left(\left|v^{a}\right|_{1, h}+\left|v^{b}\right|_{1, h}\right) .
$$

Next, we use the definition of $\mathfrak{t}$ and the mean value theorem, to obtain

$$
\begin{aligned}
\left|\mathfrak{t}_{j}\right| & \leq \sup _{\mathbb{R}}\left|\mathfrak{g}^{\prime \prime}\right| \int_{0}^{1}\left|\mathfrak{a}_{j}^{s}-\mathfrak{b}_{j}^{s}\right| \mathrm{d} s \\
& \leq \sup _{\mathbb{R}}\left|\mathfrak{g}^{\prime \prime}\right| \int_{0}^{1}\left|s\left(v_{j}^{a}-v_{j}^{b}-z_{j}^{a}+z_{j}^{b}\right)+\left(v_{j}^{b}-z_{j}^{b}\right)\right| \mathrm{d} s \\
& \leq \sup _{\mathbb{R}}\left|\mathfrak{g}^{\prime \prime}\right|\left(\left|v_{j}^{a}-v_{j}^{b}-z_{j}^{a}+z_{j}^{b}\right|+\left|v_{j}^{b}-z_{j}^{b}\right|\right), \quad j=1, \ldots, J
\end{aligned}
$$

which, leads to

$$
\|\mathfrak{t}\|_{0, h} \leq \sup _{\mathbb{R}}\left|\mathfrak{g}^{\prime \prime}\right|\left(\left\|e^{a}-e^{b}\right\|_{0, h}+\left\|e^{b}\right\|_{0, h}\right) .
$$

Finally, for $s \in[0,1]$, we apply (2.7) and (2.3), to arrive at

$$
\begin{aligned}
\left|\mathfrak{g}^{\prime}\left(\mathfrak{a}^{s}\right)-\mathfrak{g}^{\prime}\left(\mathfrak{b}^{s}\right)\right|_{1, h} & \leq \sup _{\mathbb{R}}\left|\mathfrak{g}^{\prime \prime}\right|\left|\mathfrak{a}^{s}-\mathfrak{b}^{s}\right|_{1, h}+\sup _{\mathbb{R}}\left|\mathfrak{g}^{\prime \prime \prime}\right|\left\|\delta_{h} \mathfrak{b}^{s}\right\|_{\infty, h}\left\|\mathfrak{a}^{s}-\mathfrak{b}^{s}\right\|_{0, h} \\
& \leq\left(\sup _{\mathbb{R}}\left|\mathfrak{g}^{\prime \prime}\right|+\mathrm{L} \sup _{\mathbb{R}}\left|\mathfrak{g}^{\prime \prime \prime}\right|\left\|\delta_{h} \mathfrak{b}^{s}\right\|_{\infty, h}\right)\left|\mathfrak{a}^{s}-\mathfrak{b}^{s}\right|_{1, h} \\
& \leq\left(\sup _{\mathbb{R}}\left|\mathfrak{g}^{\prime \prime}\right|+\mathrm{L} \sup _{\mathbb{R}}\left|\mathfrak{g}^{\prime \prime \prime}\right|\left\|\delta_{h} \mathfrak{b}^{s}\right\|_{\infty, h}\right)\left(\left|e^{a}-e^{b}\right|_{1, h}+\left|e^{b}\right|_{1, h}\right) .
\end{aligned}
$$


Observing that $\delta_{h} \mathfrak{t}=\int_{0}^{1} \delta_{h}\left[\mathfrak{g}^{\prime}\left(\mathfrak{a}^{s}\right)-\mathfrak{g}^{\prime}\left(\mathfrak{b}^{s}\right)\right] \mathrm{d} s$ and using (B.4) we have

$$
\begin{aligned}
|\mathfrak{t}|_{1, h} & \leq \int_{0}^{1}\left|\mathfrak{g}^{\prime}\left(\mathfrak{a}^{s}\right)-\mathfrak{g}^{\prime}\left(\mathfrak{b}^{s}\right)\right|_{1, h} \mathrm{~d} s \\
& \leq\left[\sup _{\mathbb{R}}\left|\mathfrak{g}^{\prime \prime}\right|+\mathrm{L} \sup _{\mathbb{R}}\left|\mathfrak{g}^{\prime \prime \prime}\right|\left(\left\|\delta_{h} z^{a}\right\|_{\infty, h}+\left\|\delta_{h} z^{b}\right\|_{\infty, h}\right)\right]\left(\left|e^{a}-e^{b}\right|_{1, h}+\left|e^{b}\right|_{1, h}\right) .
\end{aligned}
$$

Part II. Using the mean value theorem, we obtain

$$
\mathfrak{g}\left(v^{a}\right)-\mathfrak{g}\left(v^{b}\right)-\mathfrak{g}\left(z^{a}\right)+\mathfrak{g}\left(z^{b}\right)=\mathfrak{L}^{A}+\mathfrak{L}^{B},
$$

where $\mathfrak{L}^{A}, \mathfrak{L}^{B} \in \mathrm{X}_{h}^{\circ}$ are defined by $\mathfrak{L}^{A}:=\left(v^{a}-v^{b}-z^{a}+z^{b}\right) \otimes \mathfrak{f}$ and $\mathfrak{L}^{B}:=\left(z^{a}-z^{b}\right) \otimes \mathfrak{t}$. Thus, using (B.1) and (B.3), we have

$$
\begin{aligned}
& \left\|\mathfrak{L}^{A}\right\|_{0, h} \leq \sup _{\mathbb{R}}\left|\mathfrak{g}^{\prime}\right|\left\|e^{a}-e^{b}\right\|_{0, h}, \\
& \left\|\mathfrak{L}^{B}\right\|_{0, h} \leq \sup _{\mathbb{R}}\left|\mathfrak{g}^{\prime \prime}\right|\left|z^{a}-z^{b}\right|_{\infty, h}\left(\left\|e^{a}-e^{b}\right\|_{0, h}+\left\|e^{b}\right\|_{0, h}\right) .
\end{aligned}
$$

The desired inequality (2.8) follows, easily, as a simple outcome of (B.6) and (B.7).

Part III. For the discrete derivative of $\mathfrak{L}^{A}$ and $\mathfrak{L}^{B}$, we, easily, obtain the following formulas:

$$
\begin{aligned}
& \left(\delta_{h} \mathfrak{L}^{A}\right)_{j}=\delta_{h}\left(v^{a}-v^{b}-z^{a}+z^{b}\right)_{j} \mathfrak{f}_{j+1}+\left(v_{j}^{a}-v_{j}^{b}-z_{j}^{a}+z_{j}^{b}\right)\left(\delta_{h} \mathfrak{f}\right)_{j}, \\
& \left(\delta_{h} \mathfrak{L}^{B}\right)_{j}=\delta_{h}\left(z^{a}-z^{b}\right)_{j} \mathfrak{t}_{j+1}+\left(z^{a}-z^{b}\right)_{j}\left(\delta_{h} \mathfrak{t}\right)_{j}
\end{aligned}
$$

for $j=0, \ldots, J$, which yield

$$
\begin{aligned}
& \left|\mathfrak{L}^{A}\right|_{1, h} \leq\left|e^{a}-e^{b}\right|_{1, h}|\mathfrak{f}|_{\infty, h}+\left|e^{a}-e^{b}\right|_{\infty, h}|\mathfrak{f}|_{1, h}, \\
& \left|\mathfrak{L}^{B}\right|_{1, h} \leq\left\|\delta_{h}\left(z^{a}-z^{b}\right)\right\|\left\|_{\infty, h}\right\| \mathfrak{t} \|_{0, h}+\left|z^{a}-z^{b}\right|_{\infty, h}|\mathfrak{t}|_{1, h} .
\end{aligned}
$$

Using (B.8), (2.2), (B.1) and (B.2), we have

$$
\left|\mathfrak{L}^{A}\right|_{1, h} \leq\left[\sup _{\mathbb{R}}\left|\mathfrak{g}^{\prime}\right|+\frac{\mathrm{L}^{1 / 2}}{2} \sup _{\mathbb{R}}\left|\mathfrak{g}^{\prime \prime}\right|\left(\left|v^{a}\right|_{1, h}+\left|v^{b}\right|_{1, h}\right)\right]\left|e^{a}-e^{b}\right|_{1, h} .
$$

Combining (B.8), (B.3) and (B.5), we arrive at

$$
\begin{aligned}
\left|\mathfrak{L}^{B}\right|_{1, h} \leq & \sup _{\mathbb{R}}\left|\mathfrak{g}^{\prime \prime}\right|\left\|\delta_{h}\left(z^{a}-z^{b}\right)\right\|_{\infty, h}\left(\left\|e^{a}-e^{b}\right\|_{0, h}+\left\|e^{b}\right\|_{0, h}\right) \\
& +\left|z^{a}-z^{b}\right|_{\infty, h}\left[\sup _{\mathbb{R}}\left|\mathfrak{g}^{\prime \prime}\right|+\mathrm{L} \sup _{\mathbb{R}}\left|\mathfrak{g}^{\prime \prime \prime}\right|\left(\left\|\delta_{h} z^{a}\right\|_{\infty, h}+\left\|\delta_{h} z^{b}\right\|_{\infty, h}\right)\right] \quad\left(\left|e^{a}-e^{b}\right|_{1, h}+\left|e^{b}\right|_{1, h}\right) .
\end{aligned}
$$

Finally, (2.9) follows, easily, in view of (B.6), (B.9) and (B.10).

Acknowledgements. The author would like to thank the referees for their helpful comments and suggestions.

\section{REFERENCES}

[1] G.D. Akrivis, Finite difference discretization of the cubic Schrödinger equation. IMA J. Numer. Anal. 13 (1993) $115-124$.

[2] G.D. Akrivis and V.A. Dougalis, Finite difference discretizations of some initial and boundary value problems with interface. Math. Comput. 56 (1991) 505-522.

[3] X. Antoine, W. Bao and C. Besse, Computational methods for the dynamics of the nonlinear Schrödinger/Gross-Pitaevskii equations. Comput. Phys. Commun. 184 (2013) 2621-2633. 
[4] C. Besse, Schéma de relaxation pour l' équation de Schrödinger non linéaire et les systèmes de Davey et Stewartson. C. R. Acad. Sci. Paris Sér. I 326 (1998) 1427-1432.

[5] C. Besse, A relaxation scheme for the nonlinear Schrödinger equation. SIAM J. Numer. Anal. 42 (2004) 934-952.

[6] C. Besse, S. Descombes, G. Dujardin and I. Lacroix-Violet, Energy preserving methods for nonlinear Schrödinger equations. Preprint arXiv: 1812.04890 (2018).

[7] J.J. Dongarra, J.R. Bunch, C.B. Moller and G.W. Stewart, LINPACK Users' Guide. SIAM (1987).

[8] P. Henning and J. Wärnegård, Numerical comparison of mass-conservative schemes for the Gross-Pitaevskii equation. Kinet. Relat. Models 12 (2019) 1247-1271.

[9] O. Karakashian and Ch. Makridakis, A space-time finite element method for the nonlinear Schrödinger equation: the discontinuous Galerkin method. Math. Comput. 67 (1998) 479-499.

[10] T. Katsaounis and I. Kyza, A posteriori error analysis for evolution nonlinear Schrödinger equations up to the critical exponent. SIAM J. Numer. Anal. 56 (2018) 1405-1434.

[11] T. Katsaounis and D. Mitsotakis, On the reflection of solitons of the cubic nonlinear Schrödinger equation. Math. Methods Appl. Sci. 41 (2018) 1013-1018.

[12] O.A. Ladyzhenskaja, V.A. Solonnikov and N.N. Ural'ceva, Linear and Quasi-linear Equations of Parabolic Type. Translations of Mathematical Monographs. AMS 23 (1968).

[13] M. Li, C. Huang and W. Ming, A relaxation-type Galerkin FEM for nonlinear fractional Schrödinger equations. Numerical Algorithms 83 (2020) 99-124.

[14] D. Oelz and S. Trabelsi, Analysis of a relaxation scheme for a nonlinear Schrödinger equation occurring in Plasma Physics. Math. Modell. Anal. 19 (2014), 257-274.

[15] G.E. Zouraris, On the convergence of a linear two-step finite element method for the nonlinear Schrödinger equation. Math. Model. Numer. Anal. 35 (2001) 389-405.

[16] G.E. Zouraris, Error estimation of the Besse Relaxation Scheme for a semilinear heat equation. Preprint arXiv:1812.09273 (2018).

[17] G.E. Zouraris, Error estimation of the Relaxation Finite Difference Scheme for the nonlinear Schrödinger equation. Preprint arXiv:2002.09605 (2020).

[18] G.E. Zouraris, A Relaxation/Finite Difference discretization of a 2D Semilinear Heat Equation over a rectangular domain. Preprint arXiv:2006.14092 (2020). 